Research Article

\title{
Nonproportional Intentionally Mistuned Turbine Blisk Design with Improved Component Modal Synthesis
}

\author{
Bin Bai $\mathbb{D},{ }^{1,2,3,4}$ Qi Yang, ${ }^{1,2}$ Guang Wei Zhu, ${ }^{1,2}$ Qi Liang $W u,{ }^{5}$ and Xin ye Li $\mathbb{D}^{2}$ \\ ${ }^{1}$ State Key Laboratory of Reliability and Intelligence of Electrical Equipment, Hebei University of Technology, \\ Tianjin 300401, China \\ ${ }^{2}$ School of Mechanical Engineering, Hebei University of Technology, Tianjin 300401, China \\ ${ }^{3}$ National Engineering Research Center for Technological Innovation Method and Tool, Tianjin 300401, China \\ ${ }^{4}$ Science and Technology on Particle Transport Separation Laboratory, Institute of Physical and Chemical \\ Engineering of Nuclear Industry, Tianjin 300180, China \\ ${ }^{5}$ School of Electrical Engineering and Automation, Tiangong University, Tianjin 300387, China
}

Correspondence should be addressed to Bin Bai; baibin@126.com and Xin ye Li; xylihebut@163.com

Received 25 November 2020; Revised 23 December 2020; Accepted 31 December 2020; Published 30 January 2021

Academic Editor: Wei He

Copyright (C) 2021 Bin Bai et al. This is an open access article distributed under the Creative Commons Attribution License, which permits unrestricted use, distribution, and reproduction in any medium, provided the original work is properly cited.

\begin{abstract}
An improved component modal synthesis-based nonproportional mistuning method (ICMS-NPMM) is proposed to investigate mistuned turbine blisks (MTBs) since the high-fidelity finite element models (HFEMs) involve large number of computations, which leads to low calculation efficiency. To reduce degrees of freedom and suppress the flutter of MTB, it is divided into mistuned blade structure and tuned disk structure, and the intentional mistuning is considered. Furthermore, the mistuned parameters, nonproportional mistuning, and complex loads are also considered. Firstly, the basic theory of ICMS-NPMM is investigated; secondly, the model of MTB is established via ICMS-NPMM; finally, the intentionally mistuned design of modal shape amplitudes (MSAs) is investigated via ICMS-NPMM. The results indicate that the calculation efficiency is enhanced via ICMS-NPMM relative to that of via HFEM. In addition, the sensitivity and the flutter are decreased; meanwhile, the amplitude fluctuations of MSAs are distinctly decreased and become comparatively smooth. This investigation provides an important guidance for the vibration characteristic study of complex mechanical structures in engineering practice.
\end{abstract}

\section{Introduction}

The turbine blisks work in complex environment and bear complicated loads such as centrifugal force and thermal shock. Mistuning induces vibration and reduces the service life of turbine blisks. Besides, vibration causes the reduction of reliability and durability for turbine blisks. Moreover, the mistuning of turbine blisks is difficult to be identified and predicted. It is necessary to perform the design of mistuned turbine blisks (MTBs) to ensure the performance of aeroengine. The mistuned blisks have been researched such as vibration performance, reliability, and optimization. [1-3].

In general, the performance of mistuned blisks is affiliated to the frequency and output. For instance, the influence of mistuning on the blade-disk coupling was investigated
[4]. The frequency, critical speeds, and Campbell diagram were studied [5]. Based on the investigation of the frequency, lots of scholars studied the response, and the forced and transient responses were conducted by establishing the sector finite element model (FEM) or lumped mass model, while these models were rarely applied to engineering [6-8].

As for the above studies, it is necessary to build the model of blisks firstly. Usually, the accuracy of blisks is related with the model. The lumped mass model was constructed to study the blisks [9], but the model is simplified severely and its precision is not high. Thus, the continuous parameter model is investigated and its precision is higher than the lumped mass model. The beams or shell was used to analyze the natural frequency or vibration response for a rotational shaft-disk-blade system or a compressor blade 
$[5,10-12]$. This model can guide significance, but it is still few applied in engineering. Accordingly, the FEM [13] was developed. However, the computational efficiency of highfidelity FEM (HFEM) is low; then, an improved method named the reduced-order model (ROM) is developed, and its efficiency enhanced markedly. Numerous researchers have studied the ROM from different perspectives to improve the calculation efficiency by reducing the degrees of freedom (DOFs) of the model. A way, each bladed disk sector regarded as a substructure, was presented to address the ROM [14]. The ROM was sublimed and devoted to small geometric and stiffness mistuning, etc. [15-17]. The above investigations reveal that the ROM can increase the calculation efficiency observably. Therefore, this method is extensively utilized in engineering practice. In this work, the ROM is improved to discuss the modal shape amplitudes of the blisks.

However, the above research studies are on the blisks with passive mistuning. Virtually, the blisks of aeroengine often generate vibration or flutter during flight, which seriously affects the service life. Thus, the investigation of flutter for mistuned blisks is significant to engineering practice. To suppress the flutter of bladed disk, the intentionally mistuned design is researched. Intrinsically, the intentionally mistuned design is a robustness control and design, which got a rapid development since the 1990s. The robustness of blisks was analyzed $[9,18,19]$. The robustness of turbocharged components was investigated [20]. Jarrett et al. [21] introduced the nonintrusive polynomial chaos into the design process for a gas turbine aeroengine to facilitate a rapid robustness analysis. Saikia and Sahu [22] studied the robustness of frequency response function of a gas turbine power plant. Mohammadi and Montazeri-Gh [23] investigated the robust performance for an industrial two-shaft gas turbine. Meanwhile, numerous researchers researched the wind turbines as well. For instance, the high extremum outof-plane loads on the outlier robustness for wind turbines were discussed [24]. Some other scholars also studied the robustness of wind turbines, such as optimization efficiency and multiobjective robust optimization [25-27]. However, the robustness design of blades [28-31] had been focused since the beginning of the 21st century. The intentionally mistuned design of blisks is performed in the recent years. For example, the intentional mistuning was introduced to reduce the forced response of blade [32-35]. In addition, some other scholars conducted the reliability and optimization of blades [36-38].

However, the above investigations on intentionally mistuned blisks only focus on the displacement modal shape amplitudes, the localization factor, or only considering blade elasticity modulus as mistuned parameter; furthermore, the above approaches have unacceptable computational efficiency. To cater the actual engineering, more parameters such as blade thickness and density besides its elasticity modulus are regarded as mistuned parameters. The stress amplitudes (SAs) and strain energy amplitudes (SEAs) are regarded as the research objectives as well as displacement amplitudes (DAs), concurrently focusing the centrifugal force and thermal load. In this case, a methodology named the improved component modal synthesis-based nonproportional mistuning method (ICMS-NPMM) is proposed to investigate the modal shape amplitudes of intentionally MTB involving DAs, SAs, and SEAs.

In the other sections, Section 2 proposes the methodology of ICMS-NPMM, including tuned disk ROM, mistuned blade ROM, improved ROM, and the synthesis of blade and disk substructures, which lays a foundation for dynamic characteristic analysis of MTB, Section 3 establishes the MTB model which supports the modal shape amplitude analysis, Section 4 investigates the intentionally mistuned design of the modal shape amplitudes for MTB, including DAs, SAs, and SEAs, and Section 5 gives conclusions.

\section{Improved Component Modal Synthesis and Reduced-Order Model}

For the complex mechanical structure such as MTB, the computational efficiency is very low for HFEM. To deal with this problem, the ROM is adopted to investigate the modal shape amplitudes of MTB. Usually, blade stiffness mistuning which is a constant parameter was considered in classical component modal synthesis (CCMS). This method is named the CCMS-based proportional mistuning method (CCMSPMM). However, the change of the modal and response is different proportion in practical engineering. Actually, mistuning parameters are constantly changing during the operation of blisks. This method is termed as the CCMSbased nonproportional mistuning method (CCMS-NPMM), which only considers the elastic modulus of blade as nonproportional mistuned parameter [39]. However, different mistuned blades have different modes and responses. Therefore, not only nonproportional stiffness mistuning but also nonproportional density and thickness mistuning are considered for different blades, which is named the improved component modal synthesis-based nonproportional mistuning method (ICMS-NPMM). And the modal shape amplitudes of MTB are analyzed by ICMS-NPMM.

Due to the influences of manufacturing error, material dispersion, installation error, and wear on MTB, the mistuning is inevitable. According to the working condition, the blade is taken as mistuned structure and disk is taken as tuned structure in this work.

2.1. Tuned Reduced-Order Model of Disk. For the substructure of tuned disks, the displacement is denoted as

$$
\mathbf{x}=\left\{\begin{array}{l}
\mathbf{x}_{\Theta} \\
\mathbf{x}_{\Pi}
\end{array}\right\}=\left[\begin{array}{ll}
\boldsymbol{\Phi}_{\Theta} & \boldsymbol{\Psi}_{\Theta} \\
\boldsymbol{\Phi}_{\Pi} & \boldsymbol{\Psi}_{\Pi}
\end{array}\right]\left\{\begin{array}{l}
\mathbf{p}_{\varphi} \\
\mathbf{p}_{\psi}
\end{array}\right\},
$$

where $\Phi$ is the truncated set of normal modes; $\Psi$ is the complete set of attachment modes; $p_{\varphi}$ and $p_{\psi}$ are, respectively, the modal coordinates of $\Phi^{s}$ and $\Psi^{s}$; $\Theta$ is the internal DOFs of disk; and $\Pi$ is the interfacial DOFs between blades and disk.

By coordinate transformation, the mass and stiffness matrices of ROM for the disk are written as 


$$
\begin{aligned}
& \boldsymbol{\mu}=\left[\begin{array}{cc}
\mathbf{I} & \boldsymbol{\Phi}^{T} \mathbf{M}^{S} \boldsymbol{\Psi} \\
\boldsymbol{\Psi}^{T} \mathbf{M} \boldsymbol{\Phi} & \boldsymbol{\Psi}^{T} \mathbf{M} \boldsymbol{\Psi}
\end{array}\right], \\
& \boldsymbol{\kappa}=\left[\begin{array}{cc}
\boldsymbol{\Lambda} & \boldsymbol{\Phi}^{T} \mathbf{K} \boldsymbol{\Psi} \\
\boldsymbol{\Psi}^{T} \mathbf{K} \boldsymbol{\Phi} & \boldsymbol{\Psi}_{\Pi}
\end{array}\right],
\end{aligned}
$$

where $\mathbf{M}$ and $\mathbf{K}$ are the mass and stiffness matrices of the tuned disk.

2.2. Mistuned Reduced-Order Model of Blade. For the mistuned blade, the displacement is described as

$$
\mathbf{x}^{\prime}=\mathbf{I} \mathbf{p}_{\psi}^{\prime}=\mathbf{p}_{\psi}^{\prime},
$$

where I is the unit matrix and $\mathbf{p}_{\psi}^{\prime}$ is the modal coordinates.

The mass and stiffness matrices are described as

$$
\begin{aligned}
& \boldsymbol{\mu}^{\prime}=\mathbf{I}^{T} \mathbf{M}^{\prime} \mathbf{I}=\mathbf{M}^{\prime}, \\
& \mathbf{K}^{\prime}=\mathbf{I}^{T} \mathbf{K}^{\prime} \mathbf{I}=\mathbf{K}^{\prime},
\end{aligned}
$$

where $\mathbf{M}$ / and $\mathbf{K}$ / are, respectively, the mass and stiffness matrices in physical coordinates and $\mu \prime$ and $\kappa \prime$ are, respectively, the mass and stiffness matrices of mistuned blade ROM.

2.3. Substructure Improvement. Only the stiffness is considered as a constant mistuned parameter on the CCMSPMM, i.e., the elastic modulus is expressed as

$$
E_{n}=E_{0}\left(1+\delta_{n}^{E}\right)
$$

where $E_{0}$ is the nominal elastic modulus of the tuned turbine blisks; $E_{n}$ is the $n$th bladed elastic modulus of MTB; and $\delta_{n}^{E}$ is the mistuned parameter of the elastic modulus for the $n$th blade, which is a constant.

However, the $\delta_{n}^{E}$ is changing with the operation of MTB on the CCMS-NPMM. To cater the practical engineering, the nonproportional mistuning of blade thickness and mass is considered as well as that of the blade elastic modulus. And this methodology is termed as ICMS-NPMM.

When the mistuning of the $i$ th blade thickness is written as

$$
\Delta d_{i}=d_{0} \cdot A_{\mathrm{int}} \cdot \sin \left(\frac{2 \pi \cdot N_{h}}{N_{b}}(i-1)\right),
$$

$d_{0}$ is the blade thickness of disk, $A_{\text {int }}$ is the mistuned amplitude, $N_{b}$ is the number of blades, and $N_{h}$ is the number of harmonics.

The $i$ th blade thickness of MTB is defined as

$$
d_{i}=d_{0}+\Delta d_{i} .
$$

The mistuning of the $i$ th blade stiffness is

$$
\Delta E_{i}=E_{0} \cdot A_{\mathrm{int}} \cdot \sin \left(\frac{2 \pi \cdot N_{h}}{N_{b}}(i-1)\right) .
$$

The ith blade elasticity modulus of MTB is defined as

$$
E_{i}=E_{0}+\Delta E_{i} .
$$

When the mistuning of the $i$ th blade density is

$$
\Delta \rho_{i}=\rho_{0} \cdot A_{\text {int }} \sin \left(\frac{2 \pi \cdot N_{h}}{N_{b}}(i-1)\right),
$$

$\rho_{0}$ is the blade density of tuned blisks.

The $i$ th blade density of MTB is defined as

$$
\rho_{i}=\rho_{0}+\Delta \rho_{i} .
$$

Three mistuned parameters are considered, which caters the real engineering.

2.4. Substructure Synthesis. To combine the disk and blades ROM, the coordinated condition is given, which is expressed as

$$
\begin{aligned}
& \mathbf{x}_{\Pi}=\mathbf{x} \prime, \\
& f_{\Pi}=f \prime .
\end{aligned}
$$

According to equations (1)-(3) and equation (12), the coordinated condition is rewritten as

$$
\mathbf{x}_{\Pi}=\Phi_{\Pi} \mathbf{p}_{\varphi}+\Psi_{\Pi} \mathbf{p}_{\Psi}=\mathbf{p}_{\Psi}=\mathbf{x} \prime
$$

The mass and stiffness matrices of MTB and their modal coordinates are expressed as

$$
\begin{aligned}
\boldsymbol{\mu}^{\text {sys }} & =\boldsymbol{\mu}+\left[\begin{array}{cc}
\boldsymbol{\Phi}_{\Pi}^{T} \mathbf{M} / \boldsymbol{\Phi}_{\Pi} & \boldsymbol{\Phi}_{\Pi}^{T} M^{\prime} \boldsymbol{\Psi}_{\Pi} \\
\boldsymbol{\Psi}_{\Pi}^{T} \mathbf{M} / \boldsymbol{\Phi}_{\Pi}^{S} & \boldsymbol{\Psi}_{\Pi}^{T} M^{\prime} \boldsymbol{\Psi}_{\Pi}
\end{array}\right] \\
& =\left[\begin{array}{cc}
\mathbf{I}+\boldsymbol{\Phi}_{\Pi}^{T} \mathbf{M} / \boldsymbol{\Phi}_{\Pi} & \boldsymbol{\Phi}^{T} \mathbf{M} \boldsymbol{\Psi}+\boldsymbol{\Phi}_{\Pi}^{T} \mathbf{M} / \boldsymbol{\Psi}_{\Pi} \\
\boldsymbol{\Psi}^{T} \mathbf{M} \boldsymbol{\Phi}+\boldsymbol{\Psi}_{\Pi}^{T} \mathbf{M} / \boldsymbol{\Phi}_{\Pi} & \boldsymbol{\Psi}^{T} \mathbf{M} \boldsymbol{\Psi}+\boldsymbol{\Psi}_{\Pi}^{T} \mathbf{M} / \boldsymbol{\Psi}_{\Pi}
\end{array}\right] \\
& =\left[\begin{array}{cc}
\mathbf{I}+\boldsymbol{\Phi}_{\Pi}^{T} \mathbf{M} / \boldsymbol{\Phi}_{\Pi} & (\boldsymbol{\Lambda})^{-1} \boldsymbol{\Phi}_{\Pi}^{T}+\boldsymbol{\Phi}_{\Pi}^{T} \mathbf{M} / \Psi_{\Pi} \\
\boldsymbol{\Phi}_{\Pi}(\boldsymbol{\Lambda})^{-1}+\boldsymbol{\Psi}_{\Pi}^{T} \mathbf{M} / \boldsymbol{\Phi}_{\Pi} & \boldsymbol{\Psi}^{T} \mathbf{M} \boldsymbol{\Psi}+\boldsymbol{\Psi}_{\Pi}^{T} \mathbf{M} / \Psi_{\Pi}
\end{array}\right],
\end{aligned}
$$

$$
\begin{aligned}
\boldsymbol{\kappa}^{\mathrm{sys}} & =\boldsymbol{\kappa}+\left[\begin{array}{cc}
\boldsymbol{\Phi}_{\Pi}^{T} \mathbf{K} / \boldsymbol{\Phi}_{\Pi} & \boldsymbol{\Phi}_{\Pi}^{T} \mathbf{K} / \Psi_{\Pi} \\
\boldsymbol{\Psi}_{\Pi}^{T} \mathbf{K} / \boldsymbol{\Phi}_{\Pi} & \boldsymbol{\Psi}_{\Pi}^{T} \mathbf{K} / \Psi_{\Pi}
\end{array}\right] \\
& =\left[\begin{array}{cc}
\boldsymbol{\Lambda}+\boldsymbol{\Phi}_{\Pi}^{T} \mathbf{K} / \boldsymbol{\Phi}_{\Pi} & \boldsymbol{\Phi}^{T} \mathbf{K} \boldsymbol{\Psi}+\boldsymbol{\Phi}_{\Pi}^{T} \mathbf{K} / \Psi_{\Pi} \\
\boldsymbol{\Psi}^{T} \mathbf{K} \boldsymbol{\Phi}+\boldsymbol{\Psi}_{\Pi}^{T} \mathbf{K} / \boldsymbol{\Phi}_{\Pi} & \boldsymbol{\Psi}_{\Pi}+\boldsymbol{\Psi}_{\Pi}^{T} \mathbf{K} / \Psi_{\Pi}
\end{array}\right] \\
& =\left[\begin{array}{cc}
\boldsymbol{\Lambda}+\boldsymbol{\Phi}_{\Pi}^{T} \mathbf{K} / \boldsymbol{\Phi}_{\Pi} & \boldsymbol{\Phi}_{\Pi}^{T}+\boldsymbol{\Phi}_{\Pi}^{T} \mathbf{K} / \Psi_{\Pi} \\
\boldsymbol{\Phi}_{\Pi}+\boldsymbol{\Psi}_{\Pi}^{T} \mathbf{K} / \boldsymbol{\Phi}_{\Pi} & \boldsymbol{\Psi}_{\Pi}+\boldsymbol{\Psi}_{\Pi}^{T} \mathbf{K} / \boldsymbol{\Psi}_{\Pi}
\end{array}\right],
\end{aligned}
$$

$$
\mathbf{p}^{\text {syn }}=\left\{\begin{array}{l}
\mathbf{p}_{\varphi} \\
\mathbf{p}_{\psi}
\end{array}\right\}
$$

where $\mathbf{p}^{\text {syn }}$ is comprehensive modal coordinate.

The intrinsic frequencies of tuned blisks are still dense after a small mistuning $(0 \%-5 \%)$, and the mistuned intrinsic frequencies can be described by a tuned intrinsic subset. 
Thus, the $\Psi$ and $p_{\Psi}$ in equations (14) and (15) can be ignored for the above ROM, and the ROM of MTB is rewritten as

$$
\begin{aligned}
& \boldsymbol{\mu}^{\text {sys }}=\left[I+\Phi_{\Pi}^{T} \mathbf{M} / \Phi_{\Pi}\right], \\
& \boldsymbol{\kappa}^{\text {sys }}=\left[\boldsymbol{\Lambda}+\Phi_{\Pi}^{T} \mathbf{K} / \Phi_{\Pi}\right], \\
& \mathbf{p}^{\text {syn }}=\mathbf{p}_{\varphi},
\end{aligned}
$$

where $\quad \mathbf{M} \prime=B \operatorname{diag}_{n-1, \ldots, N}\left[\mathbf{M}_{n}^{\prime}\right] ; \quad \mathbf{K} /=B \operatorname{diag}_{n-1, \ldots, N}\left[\mathbf{K}_{n}^{\prime}\right]$; $B$ diag[.] is block diagonal matrix; and $N$ is the number of blades.

If only the normal modal is used to describe blade vibration, the boundary displacement between blades and disk cannot be obtained; therefore, the additional boundary modal must be considered. Assume that the additional boundary modal is defined as

$$
\left[\begin{array}{c}
\Psi^{\mathrm{CB}} \\
\mathbf{I}
\end{array}\right],
$$

where $\Psi^{\mathrm{CB}}$ is the internal DOFs of blades.

The relationship of mass matrix $\mathbf{M}^{\mathrm{CB}}$ and stiffness matrix $\mathbf{K}^{\mathrm{CB}}$ are, respectively, described as

$$
\begin{aligned}
& \mathbf{J}_{m}\left(\boldsymbol{\Psi}^{\mathrm{CB}}\right)=\left[\begin{array}{c}
\boldsymbol{\Psi}^{\mathrm{CB}} \\
\mathbf{I}
\end{array}\right]^{T}\left[\begin{array}{ll}
\mathbf{M}_{i i}^{\mathrm{CB}} & \mathbf{M}_{i b}^{\mathrm{CB}} \\
\mathbf{M}_{i b}^{\mathrm{CB}} & \mathbf{M}_{b b}^{\mathrm{CB}}
\end{array}\right]\left[\begin{array}{c}
\boldsymbol{\Psi}^{\mathrm{CB}} \\
\mathbf{I}
\end{array}\right], \\
& \mathbf{J}_{k}\left(\boldsymbol{\Psi}^{\mathrm{CB}}\right)=\left[\begin{array}{c}
\boldsymbol{\Psi}^{\mathrm{CB}} \\
\mathbf{I}
\end{array}\right]^{T}\left[\begin{array}{ll}
\mathbf{K}_{i i}^{\mathrm{CB}} & \mathbf{K}_{i b}^{\mathrm{CB}} \\
\mathbf{K}_{i b}^{\mathrm{CB}} & \mathbf{K}_{b b}^{\mathrm{CB}}
\end{array}\right]\left[\begin{array}{c}
\boldsymbol{\Psi}^{\mathrm{CB}} \\
\mathbf{I}
\end{array}\right],
\end{aligned}
$$

where $i$ and $b$ are, respectively, the internal and boundary DOFs of blades.

The relationship of the minimum boundary modal $\Psi^{\mathrm{CB}, m}$ and $\Psi^{\mathrm{CB}, k}$ is, respectively, obtained via transforming of $\mathbf{M}^{\mathrm{CB}}$ and $\mathbf{K}^{\mathrm{CB}}$ based on the first-order differential of $\mathbf{J}_{m}$ and $\mathbf{J}_{k}$, which are expressed as

$$
\begin{gathered}
\mathbf{M}_{i i}^{\mathrm{CB}} \Psi^{\mathrm{CB}, m}+\mathbf{M}_{i b}^{\mathrm{CB}}=0, \\
\mathbf{K}_{i i}^{\mathrm{CB}} \boldsymbol{\Psi}^{\mathrm{CB}, m}+\mathbf{K}_{i b}^{\mathrm{CB}}=0 .
\end{gathered}
$$

In fact, the boundary modal is the smallest which contributes kinetic and potential energy to the blades. The relationship of normal modal and boundary modal corresponding to the mass and stiffness mistuning is, respectively, expressed as

$$
\begin{aligned}
& \boldsymbol{\Phi}_{\Pi, n}^{m}=\left[\begin{array}{cc}
\boldsymbol{\Phi}^{\mathrm{CB}} & \boldsymbol{\Psi}^{\mathrm{CB}, m} \\
0 & \mathrm{I}
\end{array}\right]\left[\begin{array}{c}
\mathbf{q}_{\varphi, n}^{\mathrm{CB}, m} \\
\mathbf{q}_{\Psi, n}^{\mathrm{CB}}
\end{array}\right], \\
& \boldsymbol{\Phi}_{\Pi, n}^{k}=\left[\begin{array}{cc}
\boldsymbol{\Phi}^{\mathrm{CB}} & \boldsymbol{\Psi}^{\mathrm{CB}, k} \\
0 & \mathrm{I}
\end{array}\right]\left[\begin{array}{c}
\mathbf{q}_{\varphi, n}^{\mathrm{CB}, k} \\
\mathbf{q}_{\Psi, n}^{\mathrm{CB}}
\end{array}\right],
\end{aligned}
$$

where $\mathbf{q}_{\varphi, n}^{\mathrm{CB}, m}, \mathbf{q}_{\Psi, n}^{\mathrm{CB}}$, and $\mathbf{q}_{\varphi, n}^{\mathrm{CB}, k}$ are, respectively, the $n$th bladed normal and boundary modals.

Therefore, the relationship of all blades can be expressed

$$
\begin{gathered}
\boldsymbol{\Phi}_{\Pi}^{m}=\left(\mathbf{I} \otimes \mathbf{U}^{\mathrm{CB}, m}\right) \mathbf{q}^{\mathrm{CB}, m}, \\
\boldsymbol{\Phi}_{\Pi}^{k}=\left(\mathbf{I} \otimes \mathbf{U}^{\mathrm{CB}, k}\right) \mathbf{q}^{\mathrm{CB}, k},
\end{gathered}
$$

where

$$
\begin{aligned}
& \mathbf{U}^{\mathrm{CB}, m}=\left[\begin{array}{cc}
\boldsymbol{\Phi}^{\mathrm{CB}} & \boldsymbol{\Psi}^{\mathrm{CB}, m} \\
0 & \mathrm{I}
\end{array}\right], \\
& \mathbf{U}^{\mathrm{CB}, k}=\left[\begin{array}{cc}
\boldsymbol{\Phi}^{\mathrm{CB}} & \boldsymbol{\Psi}^{\mathrm{CB}, k} \\
0 & \mathrm{I}
\end{array}\right] \text {, } \\
& \mathbf{q}^{\mathrm{CB}, m}=\left[\begin{array}{c}
\vdots \\
\mathbf{q}_{\varphi, n}^{\mathrm{CB}, m} \\
\mathbf{q}_{\Psi, n}^{\mathrm{CB}, m} \\
\vdots
\end{array}\right] \text {, } \\
& \mathbf{q}^{\mathrm{CB}, k}=\left[\begin{array}{c}
\vdots \\
\mathbf{q}_{\varphi, n}^{\mathrm{CB}, k} \\
\mathbf{q}_{\Psi, n}^{\mathrm{CB}} \\
\vdots
\end{array}\right],
\end{aligned}
$$

where $\otimes$ is Kronecker product.

Equations (24) and (25) are substituted into equations (17) and (18), and the mass and stiffness matrices of ROM are, respectively, rewritten as

$$
\begin{aligned}
\boldsymbol{\mu}^{\mathrm{syn}} & =\mathbf{I}+\left(\mathbf{q}^{\mathrm{CB}, m}\right)^{T}\left(\mathbf{I} \otimes\left(\mathbf{U}^{\mathrm{CB}, m}\right)^{T}\right) \mathbf{M} \prime\left(\mathbf{I} \otimes \mathbf{U}^{\mathrm{CB}, m}\right)\left(\mathbf{q}^{\mathrm{CB}, m}\right) \\
& =\mathbf{I}+\underset{n=1, \ldots, N}{\mathbf{B} \operatorname{diag}}\left(\mathbf{q}_{n}^{\mathrm{CB}, m}\right)^{T}\left(\mathbf{U}^{\mathrm{CB}, m}\right)^{T} \mathbf{M}_{n}^{[\{\}]}\left(\mathbf{U}^{\mathrm{CB}, m}\right)\left(\mathbf{q}_{n}^{\mathrm{CB}, m}\right) \\
& =\mathbf{I}+\underset{n=1, \ldots, N}{\mathbf{B} \operatorname{diag}}\left(\mathbf{q}_{n}^{\mathrm{CB}, m}\right)^{T}\left[\begin{array}{cc}
\boldsymbol{\mu}_{\varphi \varphi, n}^{\prime} & \boldsymbol{\mu}_{\varphi \Psi, n}^{\prime} \\
\left(\boldsymbol{\mu}_{\varphi \Psi, n}^{\prime}\right)^{T} & \boldsymbol{\mu}_{\Psi \Psi, n}^{\prime}
\end{array}\right]\left(\mathbf{q}^{\mathrm{CB}, m}\right), \\
& \mathbf{\kappa}^{\text {syn }}=\boldsymbol{\Lambda}+\left(\mathbf{q}^{\mathrm{CB}, k}\right)^{T}\left(\mathbf{I} \otimes\left(\mathbf{U}^{\mathrm{CB}, k}\right)^{T}\right) \mathbf{M} \prime\left(\mathbf{I} \otimes \mathbf{U}^{\mathrm{CB}, k}\right)\left(\mathbf{q}^{\mathrm{CB}, k}\right) \\
& =\boldsymbol{\Lambda}+\underset{n=1, \ldots, N}{\mathbf{B} \operatorname{diag}}\left(\mathbf{q}_{n}^{\mathrm{CB}, k}\right)^{T}\left(\mathbf{U}^{\mathrm{CB}, k}\right)^{T} \mathbf{M}_{n}^{\prime}\left(\mathbf{U}^{\mathrm{CB}, k}\right)\left(\mathbf{q}_{n}^{\mathrm{CB}, k}\right) \\
& =\boldsymbol{\Lambda}+\underset{n=1, \ldots, N}{\mathbf{B} \operatorname{diag}}\left(\mathbf{q}_{n}^{\mathrm{CB}, k}\right)^{T}\left[\begin{array}{cc}
\mathbf{\kappa}_{\varphi \varphi, n}^{\prime} & \mathbf{\kappa}_{\varphi \Psi, n}^{\prime} \\
\left(\boldsymbol{\kappa}_{\varphi \Psi, n}^{\prime}\right)^{T} & \boldsymbol{\kappa}_{\Psi \Psi, n}^{\prime}
\end{array}\right]\left(\mathbf{q}_{n}^{\mathrm{CB}, k}\right),
\end{aligned}
$$

where

$$
\begin{aligned}
& \boldsymbol{\mu}_{\varphi \varphi, n}^{\prime}=\left(\boldsymbol{\Phi}^{\mathrm{CB}}\right)^{T} \mathbf{M}_{i i, n}{ }^{\prime} \boldsymbol{\Phi}^{\mathrm{CB}}+\boldsymbol{\mu}_{\varphi \Psi, n}{ }^{\prime}=\left(\boldsymbol{\Phi}^{\mathrm{CB}}\right)^{T}\left[\mathbf{M}_{i i, n}{ }^{\prime} \boldsymbol{\Psi}^{\mathrm{CB}, m}+\mathbf{M}_{i b, n}{ }^{\prime}\right], \\
& \boldsymbol{\mu}_{\Psi \Psi, n}^{\prime}=\left(\boldsymbol{\Psi}^{\mathrm{CB}, m}\right)^{T}\left[\mathbf{M}_{i i, n}{ }^{\prime} \boldsymbol{\Psi}^{\mathrm{CB}, m}+\mathbf{M}_{i b, n}{ }^{\prime}\right]+\left(\mathbf{M}_{i b, n}{ }^{\prime}\right)^{T} \boldsymbol{\Psi}^{\mathrm{CB}, m}+\mathbf{M}_{b b, n}{ }^{\prime}, \\
& \boldsymbol{\kappa}_{\varphi \varphi, n}^{\prime}=\left(\boldsymbol{\Phi}^{\mathrm{CB}}\right)^{T} \mathbf{K}_{i i, n}{ }^{\prime} \boldsymbol{\Phi}^{\mathrm{CB}}+\boldsymbol{\kappa}_{\varphi \Psi, n}{ }^{\prime}=\left(\boldsymbol{\Phi}^{\mathrm{CB}}\right)^{T}\left[\mathbf{K}_{i i, n}{ }^{\prime} \boldsymbol{\Psi}^{\mathrm{CB}, k}+\mathbf{K}_{i b, n}{ }^{\prime}\right] \text {, } \\
& \boldsymbol{K}_{\Psi \Psi, n}{ }^{\prime}=\left(\boldsymbol{\Psi}^{\mathrm{CB}, k}\right)^{T}\left[\mathbf{K}_{i i, n}{ }^{\prime} \boldsymbol{\Psi}^{\mathrm{CB}, k}+\mathbf{K}_{i b, n}{ }^{\prime}\right]+\left(\mathbf{K}_{i b, n}{ }^{\prime}\right)^{T} \boldsymbol{\Psi}^{\mathrm{CB}, k}+\mathbf{K}_{b b, n}{ }^{\prime} .
\end{aligned}
$$


Equations (27) and (28) can be used in various mistuned blisks, but mistuned value associated with boundary modal needs to be known; however, it is almost impossible to achieve. Therefore, assuming that the boundary displacement of the normal modal is smaller, or there is no mistuning of boundary unit, i.e., the kinetic and potential energy of boundary displacement is ignored. In this case, the mass and stiffness matrices of ROM for MTB can be expressed as

$$
\begin{gathered}
\boldsymbol{\mu}^{\mathrm{syn}} \approx \mathbf{I}+\underset{n=1, \ldots, N}{\mathbf{B d i a g}}\left[\left(\mathbf{q}_{\varphi, n}^{\mathrm{CB}, m}\right)^{T} \boldsymbol{\mu}_{\varphi \varphi, n} \mathbf{q}_{\varphi, n}^{\mathrm{CB}, m}\right] \\
\boldsymbol{\kappa}^{\mathrm{syn}} \approx \boldsymbol{\Lambda}+\underset{n=1, \ldots, N}{\mathbf{B d i a g}}\left[\left(\begin{array}{lll}
\left(\mathbf{q}_{\varphi, n}^{\mathrm{CB}, k}\right)^{T} & \boldsymbol{\kappa}_{\varphi \varphi, n}^{\prime} & \mathbf{q}_{\varphi, n}^{\mathrm{CB}, k}
\end{array}\right] .\right.
\end{gathered}
$$

This ROM only has blade normal modal, and the kinetic and potential energy of boundary displacement is ignored. This model can be used to analyze the maximum modal shape amplitudes of MTB.

\section{Modeling}

MTB modeling is the foundation for investigating the DAs, SAs, and SEAs. Thus, the model parameters need to be given at first, and the main parameters of MTB are shown in Table 1. The variations of blade stiffness, density, and thickness are shown in Figures 1 3.

The interfaces between blades and disk as well as the interfaces between disk hole and disk are the key joints. Therefore, only the interface DOFs are calculated, which is a huge saving in terms of computational cost. The master DOFs of the MTB are shown in Figure 4.

Only the disk is regarded as superelement [40]; however, the reduced-order FEM of MTB is established via ICMSNPMM in Figure 5.

To verify the DOFs are reduced by ISCMS, the elements are calculated as shown in Table 2.

It can be seen from Table 2 and Figure 5 that compared with classical SCMS and HFEM, ratios of decline are, respectively, $84.22 \%$ and $96.11 \%$; thus, the DOFs of MTB are distinctly reduced so that the computational time is obviously shorted relative to CCMS and HFEM.

\section{Maximum Modal Shape Amplitude Analysis}

The MTB works in severe environment, and it is very sensitive to mistuning which can trigger vibration localization even high fatigue stress and fracture. Therefore, it is very necessary to investigate the modal shape under centrifugal force and thermal load. The difference from previous studies (localization factor as research object [40])is that the modal shape amplitudes are regarded as the research object so as to make sure the variation tendency of most dangerous position and reasonably prevent MTB from destroying by mistuning. This method is simple and has high computational efficiency. To comprehensively study vibration characteristics of MTB, the DAs, SAs, and SEAs are investigated, respectively.
TABle 1: Parameters of MTB.

\begin{tabular}{lccccc}
\hline Disk & \multicolumn{3}{c}{ Blades } & \multicolumn{2}{c}{ MTB } \\
\hline$\rho_{d} \% \times 10^{3}\left(\mathrm{~kg} / \mathrm{m}^{3}\right)$ & 8.560 & $\operatorname{den}_{b} \% \times 10^{3}\left(\mathrm{~kg} / \mathrm{m}^{3}\right)$ & 8.249 & $w$ & 1120 \\
$e_{d} \% \times 10^{11}(\mathrm{~Pa})$ & 1.841 & $e_{b} \% \times 10^{11}(\mathrm{~Pa})$ & 1.7823 & $t$ & 1100 \\
$p_{d}$ & 0.3143 & $p_{b}$ & 0.3181 & $h$ & 3.0 \\
$\alpha_{d} \% \times 10^{-5}\left({ }^{\circ} \mathrm{C}^{-1}\right)$ & 1.216 & $\alpha_{b} \% \times 10^{-5}\left({ }^{\circ} \mathrm{C}^{-1}\right)$ & 1.268 & - & - \\
$k_{d} \% \mathrm{~W}\left(\mathrm{~m} .{ }^{\circ} \mathrm{C}\right)-1$ & 27.21 & $k_{b} \% \mathrm{~W}\left(\mathrm{~m} .{ }^{\circ} \mathrm{C}\right)$ & 29.72 & - & - \\
\hline
\end{tabular}

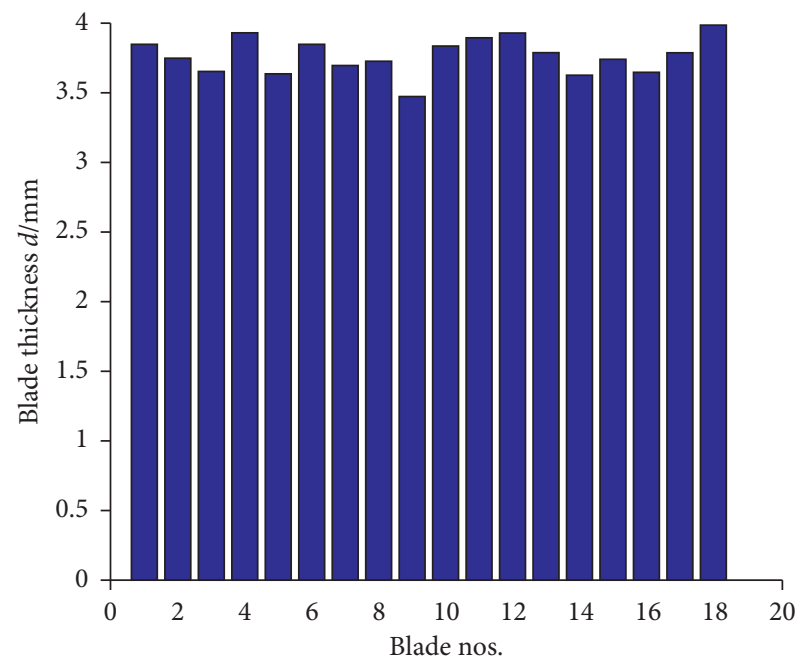

Figure 1: Blade thickness.

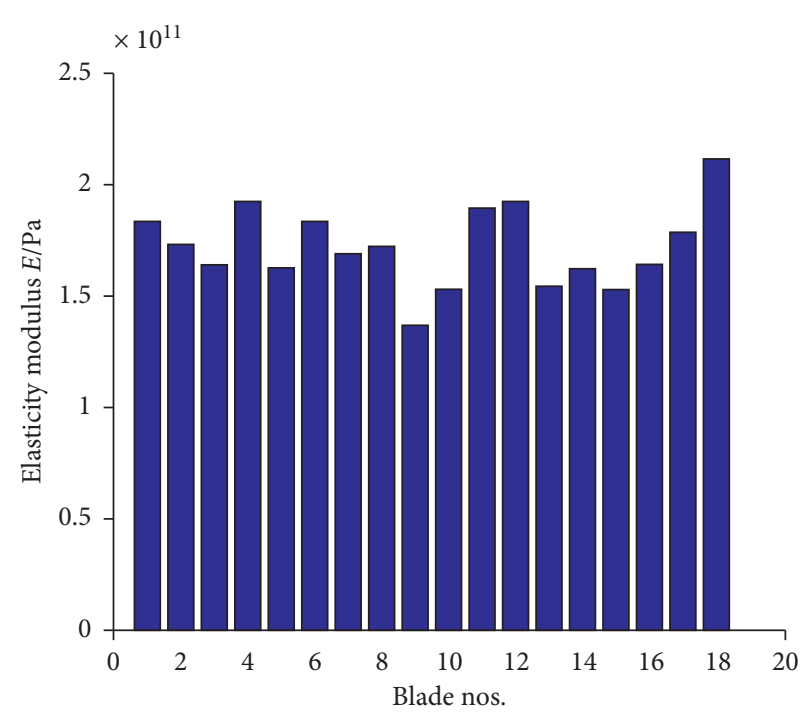

FIgURE 2: Blade elasticity modulus.

4.1. Verification. The investigation indicates that the one vibration period of blisks is about 23-24-order frequencies, and the blisks are mainly affected by the low-order modes. Meanwhile, in order to improve the calculation efficiency, the first two vibration period modes are studied. Thus, the first 38 intrinsic frequencies of MTB with $w=1120 \mathrm{rad} \cdot \mathrm{s}^{-1}$, $t=1100^{\circ} \mathrm{C}$ are discussed by ICMS-NPMM in Figure 6 to verify the effectiveness and rationality of ICMS-NPMM. The first 10, 20, 30, and 38 intrinsic frequencies are, respectively, 


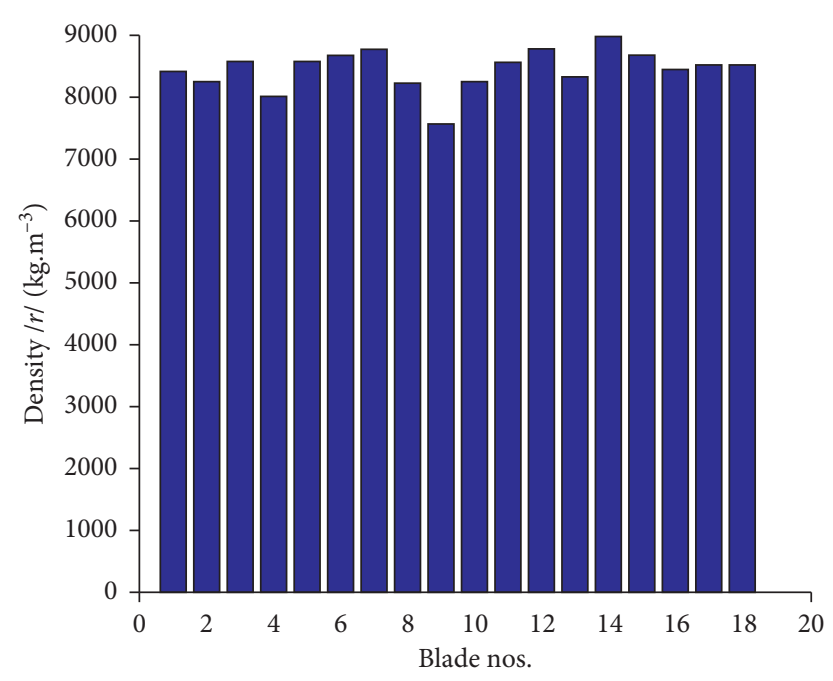

Figure 3: Blade density.

calculated by ICMS-NPMM, CCMS-NPMM, and HFEM in Figure 7 and Table 3.

Figure 6 indicates that the errors of inherent frequencies for MTB are $0.004 \% \sim 0.102 \%$ and $0.006 \% \sim 0.159 \%$ by ICMSNPMM and CCMS-NPMM relative to that of HFEM. The inherent frequencies acquired by ICMS-NPMM and CCMSNPMM are practical unanimity with that of HFEM. Thus, the accuracy of SCMS-NPMM and CCMS-NPMM is comparable to that of HFEM. Meanwhile, the computational time and saving ratio are given in Table 3 and Figure 7 . The computational time of MTB is, respectively, reduced by $7.51 \% \sim 29.13 \%$ and $10.48 \% \sim 49.15 \%$ with CCMS-NPMM and ICMS-NPMM relative to HFEM. It is observed that the larger of the orders, the higher of the time saving ratio. Thus, the calculative time of ICMS-NPMM is lower than that of CCMS-NPMM, which indicates that the ICMS-NPMM is superior to CCMS-NPMM. Also, it can be seen from Table 3 that the time-saving rate is higher with the rise of the frequency order and the main reason is that the energy of the blade disk cannot be uniformly transmitted out due to the existence of mistuning. The accumulated energy increases with the improvement of order so as to the calculation time becomes slower and slower for the HFEM. However, ICMSNPMM proposed solved the problem.

4.2. Modal Shape Amplitudes. The MTB is subjected to common action of centrifugal force and thermal load; therefore, it is necessary to investigate the intrinsic shape amplitudes. To study the common effect of centrifugal force and thermal load on the modal shape amplitudes, the DAs, SAs, and SEAs of the tuned and mistuned blisks are researched under interaction of centrifugal force and thermal load. The first 38 order DAs, SAs, and SEAs, are respectively, calculated using ICMS-NPMM, as shown in Figures 8(a), 9(a), and 10(a); the deviations of DAs, SAs, and SEAs are shown in Figures 8(b), 9(b), and 10(b); the increments of DAs, SAs, and SEAs and their comparison are shown in Figures 8(c), 9(c), and 10(c). The explanation of Figures $8-10$ is shown in Table 4 .
As seen in Figures 8 10, the variation tendencies of DAs, SAs, and SEAs under the combined action with speeds and temperatures are analogical to those of only speeds or temperatures considered; however, there are some differences.

Figure 8 indicates that the DAs are closer to those of only speeds considered with the combined action of speeds and temperatures, and the research indicates that DAs are more effected by speed than temperature. Besides, the change trend of DAs is analogical to those of only speeds or temperatures taken into account and corresponding values are reduced. Also, the mistuning is more effected than the speed when the speed and temperature simultaneously act in MTB.

Figure 9 manifests that the instability of SAs is observed, the danger rises obviously, and the destroy of MTB increases from the 24 th order when the temperatures and speeds are both considered. The main reason is the fluctuation obviously increases from the 24 th order model. In addition, the variable quantities of SAs arise in low-order and high-order frequencies when the temperature is changeless and the speed raises, or the speed is changeless and temperature rises, which intensifies destruction of MTB.

Figure 10 shows that the SEAs are sensitive to the temperature in high-order frequency and the MTB is more likely failure from the 24th order model. Thus, the influence of temperature on the SEAs will strengthen when the temperature enhances to $1100^{\circ} \mathrm{C}$. In addition, the deviations of SEAs increase sharply in the first vibration period. Distinctly, the destructive of MTB increases when the rotational speed is invariant and temperature is enhanced to a certain degree. Meanwhile, the SEAs in the 9th order frequency occur larger fluctuation when the temperature is changeless but speed is varied. Therefore, the SEAs are impacted by speed in low-order frequency. This investigation indicates that the effect of temperature on SEAs in high-order frequency is larger. The increment of SEAs is a bit more complicated, and the fluctuation is observed in low-order frequency. 


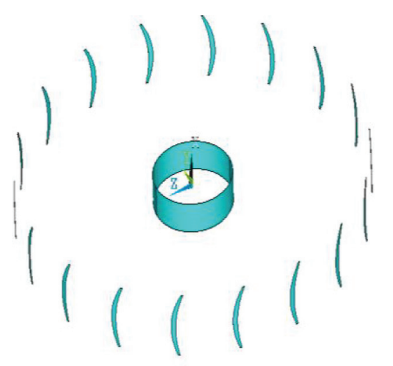

(a)

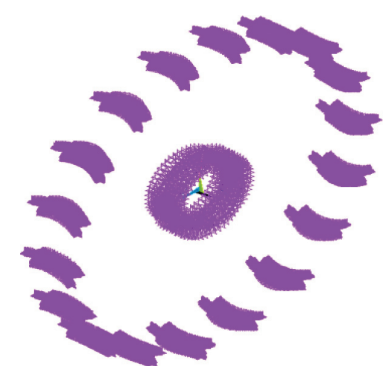

(b)

Figure 4: Master DOFs of the MTB. (a) Interfaces of master DOFs. (b) Master DOFs.

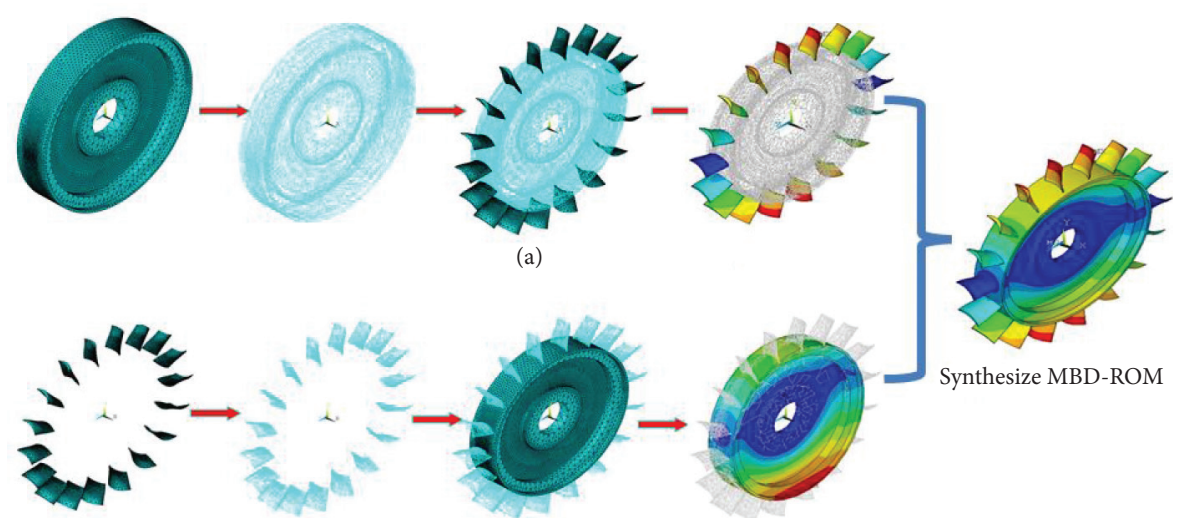

(b)

Figure 5: Reduced-order FEM of MTB. (a) Disk cohesion and blade substructure. (b) Blade cohesion and disk substructure.

TABle 2: The elements of MTB.

\begin{tabular}{lccccc}
\hline-- & HFEM & Classical SCMS & Ratio of decline (\%) & ISCMS & Ratio of decline (\%) \\
\hline Element nos. & 1102519 & 173911 & 84.22 & 42998 & 96.11 \\
\hline
\end{tabular}

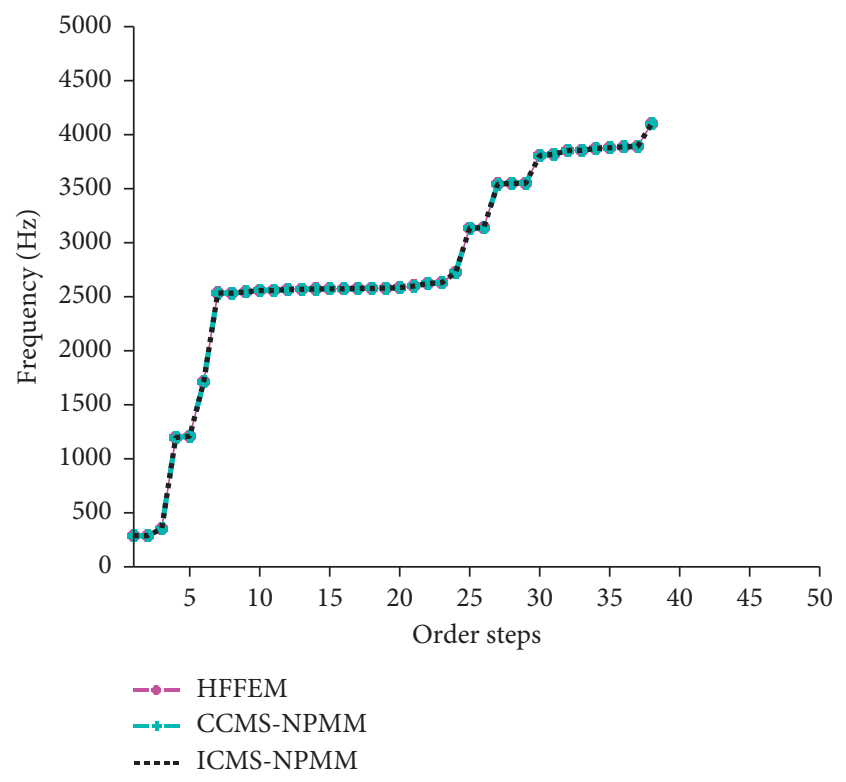

FIGURE 6: Intrinsic frequency analysis.

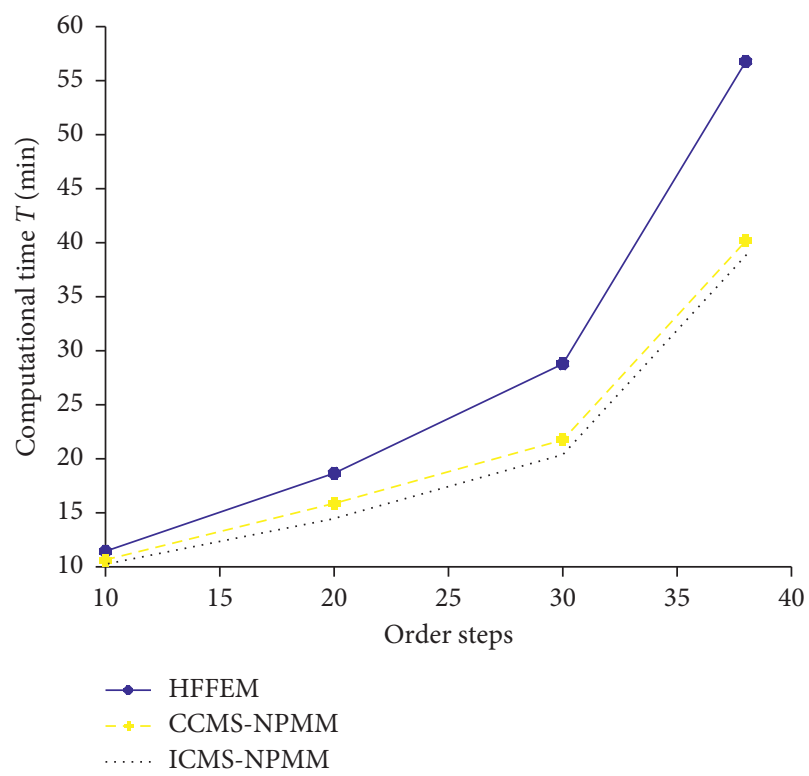

FIgURE 7: Computational time of different order frequencies. 
TABLE 3: Computational time and time saving ratio of three methods.

\begin{tabular}{lccrr}
\hline First $n$ orders & HFEM $t$ & CCMS-NPMM $t \gamma(\%)$ & ICMS-NPMM $t \gamma(\%)$ \\
\hline 10 & 11.45 & 10.59 & 7.51 & 10.25 \\
20 & 18.67 & 15.89 & 14.89 & 14.47 \\
30 & 28.79 & 21.75 & 24.45 & 18.39 \\
38 & 56.78 & 40.24 & 29.13 & 28.47 \\
\hline
\end{tabular}

Note: $t$ is the computational time of different order frequencies; $\gamma$ is the time saving ratio for CCMS-NPMM and ICMS-NPMM relative to HFEM.

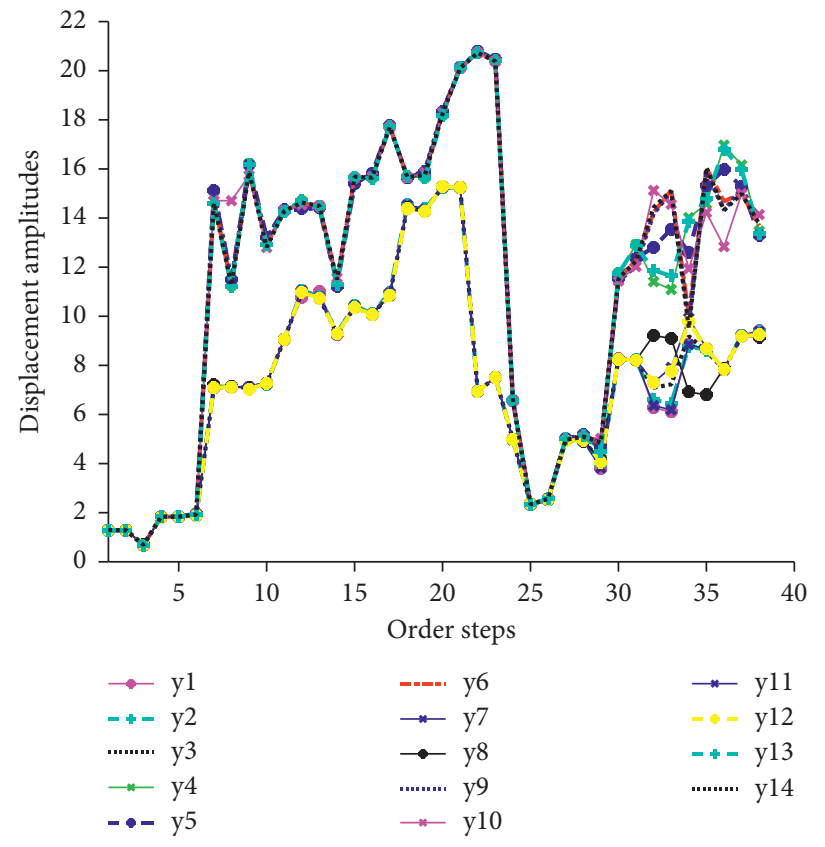

(a)
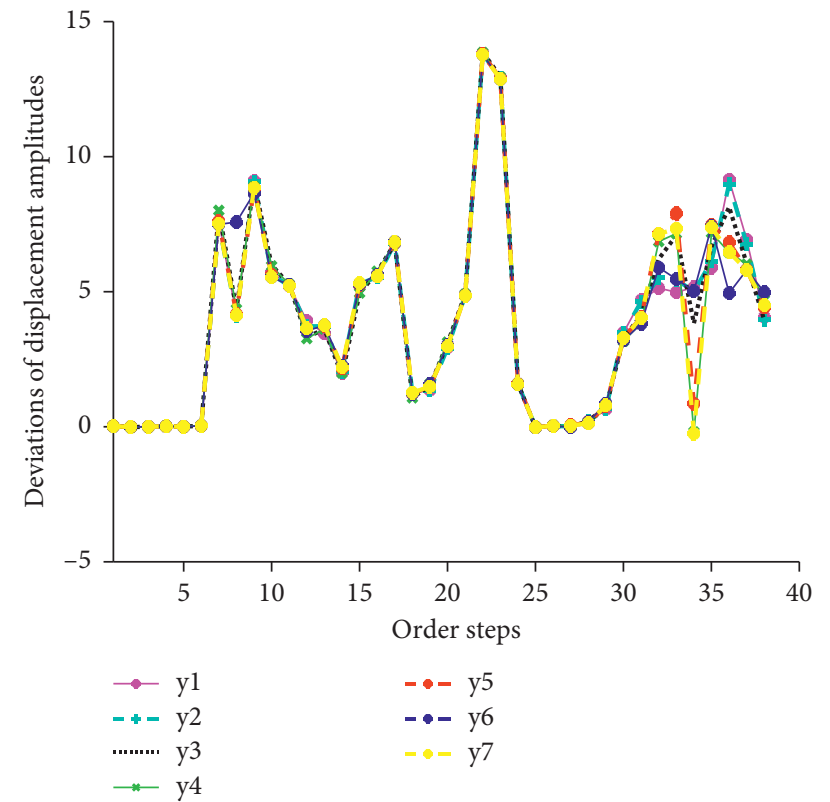

(b)

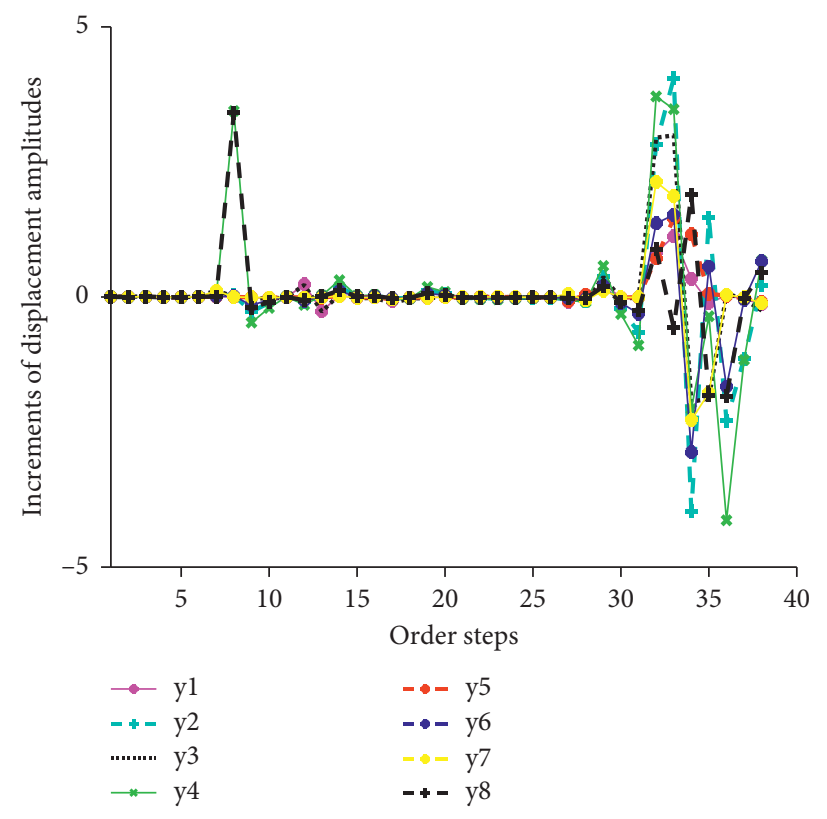

(c)

FIGURE 8: Displacement amplitudes with centrifugal force and thermal load. (a) Das. (b) Deviation of Das. (c) Variable quantity of DAs. 


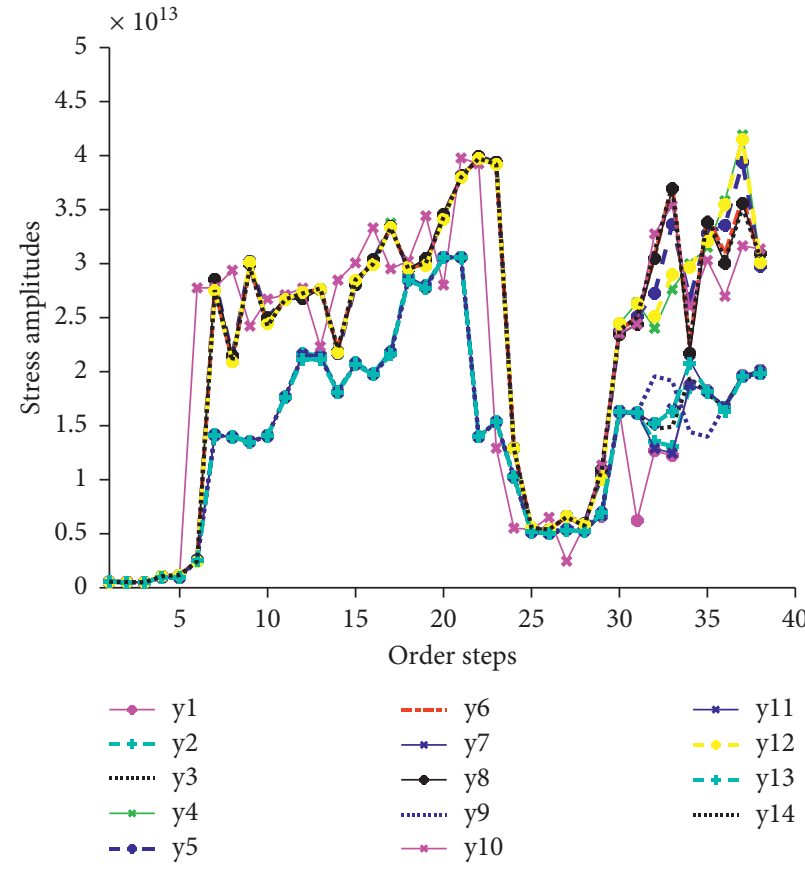

(a)

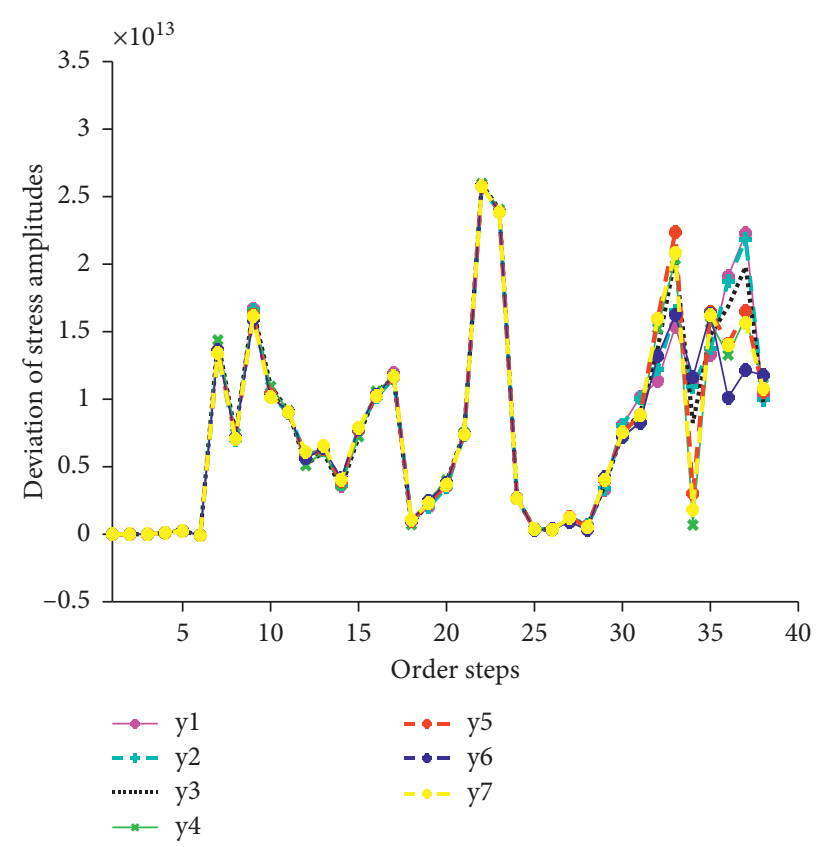

(b)



(c)

FIGURE 9: Stress amplitudes with centrifugal force and thermal load. (a) SAs. (b) Deviation of SAs. (c) Variable quantity of SAs.

4.3. Intentionally Mistuned Design. Section 4.2 manifests the localization of the modal shape amplitude caused by mistuning remarkably increases and exacerbates MTB compared with the TTB. This can induce flutter which can cause catastrophic damage to MTB, especially, to blades. In fact, the aeroengine will fluctuate or vibrate seriously due to the disturbance of air flow during the flight of aircraft, and the installation error or wear will result passive mistuning, which will induce cracks failure or fatigue fracture for blades. The intentionally mistuned design can reduce passive mistuning. To suppress the flutter, the intentionally mistuned design is investigated for MTB, this design can improve the safety and prolong its service life.

To decline the flutter of mistuned blisks, it is vital to find the most influential factors on the structural characteristics and the best combination. The mainly influence factors are shown in Figure 11 by sensitivity analysis [41]. However, the severe mistuning can cause intensive vibration of blades. The experimental study indicates that the density and elastic modulus of each three blades are the same, which can reduce 


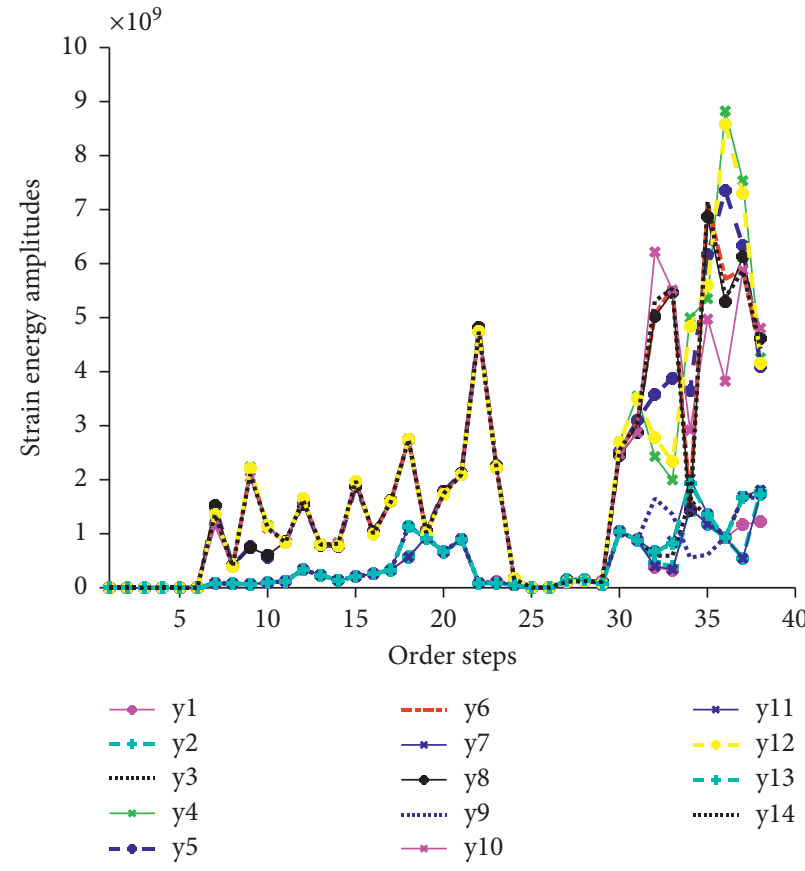

(a)
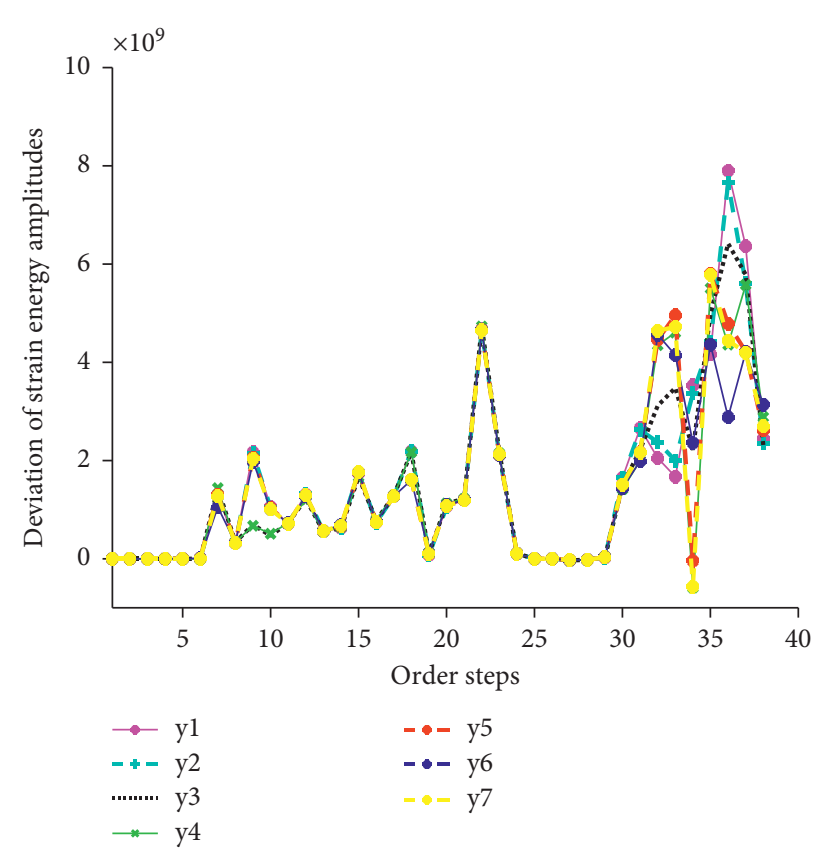

(b)

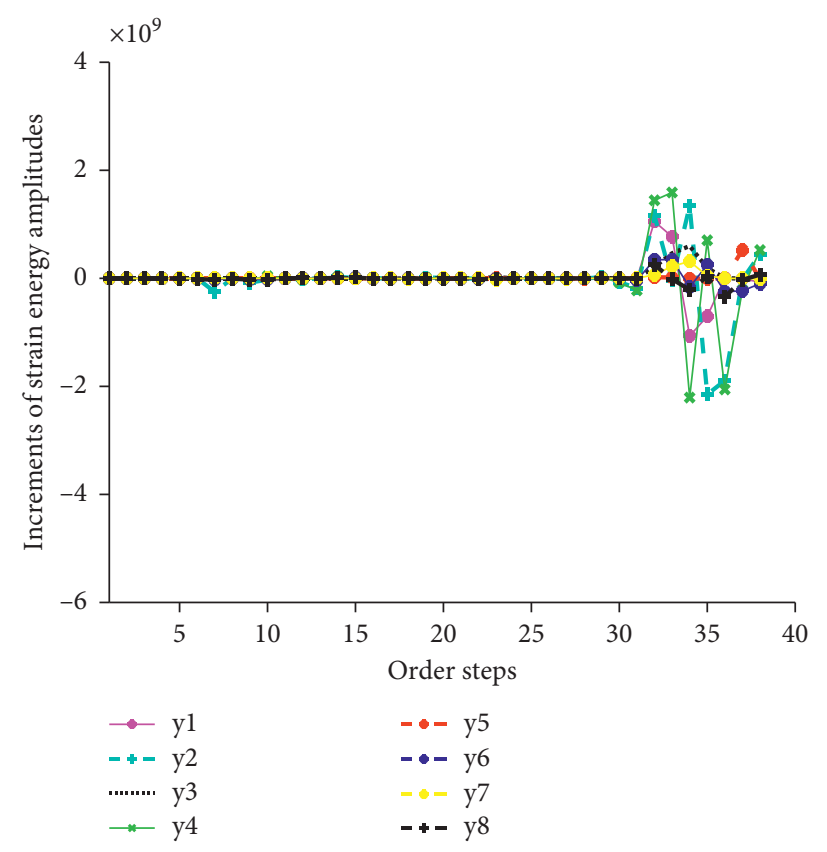

(c)

FIGURE 10: Strain energy amplitudes with centrifugal force and thermal load. (a) SEAs. (b) Deviation of SEAs. (c) Variable quantity of SEAs.

the passive mistuning. Thus, the elastic modulus and density of blades can be reasonably designed to improve the robustness and reduce the flutter of MTB as well as decline its fluctuation, which are shown in Figure 12.

Then, the DAs, SAs, and SEAs of intentional MTB are investigated via ICMS-NPMM, and the results are drawn in Figures 13 15.

Figures 13(a) 15(a) manifest that the fluctuations of DAs, SAs, and SEAs become smooth and steady in the first vibration period and distinctly decline in the second vibration period for intentionally MTB compared with passive MTB, which means the stability and safety enhances of blisks; meanwhile, it is observed that their variation tendencies of intentional mistuning are similar to those of tuning and passive mistuning, but the values are less than those of passive mistuning, which are significantly reduced. Figures 13(b) 15(b) indicate that the DAs, SAs, and SEAs are greatly affected by rotational speed in low-order 
TABLE 4: The explanation of Figures 8-10.

\begin{tabular}{|c|c|c|c|c|}
\hline \multicolumn{2}{|l|}{ Figures $8(a) / 9(a) / 10(a)$} & \multirow{2}{*}{$\begin{array}{c}\text { Figures } 8(\mathrm{~b}) / 9(\mathrm{~b}) / 10(\mathrm{~b}) \\
\text { Deviations of mistuned } \\
\text { blisks relative to tuned blisks }\end{array}$} & \multicolumn{2}{|l|}{ Figures $8(\mathrm{c}) / 9(\mathrm{c}) / 10(\mathrm{c})$} \\
\hline Tuned blisks & Mistuned blisks & & Tuned blisks & Mistuned blisks \\
\hline $\begin{array}{l}\mathrm{y} 1: w=1120 \mathrm{rad} \\
\mathrm{s}^{-1}\end{array}$ & $\mathrm{y} 4: w=\underset{\mathrm{s}^{-1}}{1120 \mathrm{rad}}$ & $\mathrm{y} 1: w=\underset{\mathrm{s}^{-1}}{1120 \mathrm{rad}}$ & $\begin{array}{l}\mathrm{y} 1: w=1120 \mathrm{rad} \cdot \mathrm{s}^{-1}, t=780^{\circ} \mathrm{C} \\
\quad \text { relative to } w=1120 \mathrm{rad} \cdot \mathrm{s}^{-1}\end{array}$ & $\begin{array}{c}\mathrm{y} 2: w=1120 \mathrm{rad} \cdot \mathrm{s}^{-1} \\
t=780^{\circ} \mathrm{C} \text { relative to } \\
w=1120 \mathrm{rad} \cdot \mathrm{s}^{-1}\end{array}$ \\
\hline $\mathrm{y} 2: t=780^{\circ} \mathrm{C}$ & $\mathrm{y} 5: t=780^{\circ} \mathrm{C}$ & $\mathrm{y} 2: w=\underset{\mathrm{s}^{-1}}{1250 \mathrm{rad}}$ & $\begin{array}{l}\text { y3: } w=1120 \mathrm{rad} \cdot \mathrm{s}^{-1}, t=780^{\circ} \mathrm{C} \\
\text { relative to } w=1120 \mathrm{rad} \cdot \mathrm{s}^{-1}\end{array}$ & $\begin{array}{c}\mathrm{y} 4: w=1120 \mathrm{rad} \cdot \mathrm{s}^{-1} \\
t=1100^{\circ} \mathrm{C} \text { relative to } \\
w=1120 \mathrm{rad} \cdot \mathrm{s}^{-1}\end{array}$ \\
\hline $\begin{array}{l}\mathrm{y} 3: w=1120 \mathrm{rad} \cdot \mathrm{s}^{-1} \\
t=780^{\circ} \mathrm{C}\end{array}$ & $\begin{array}{c}\mathrm{y} 6: \mathrm{w}=1120 \\
\mathrm{rad} \cdot \mathrm{s}^{-1} \\
t=780^{\circ} \mathrm{C}\end{array}$ & $\mathrm{y} 3: t=780^{\circ} \mathrm{C}$ & y5: $t=1100^{\circ} \mathrm{C}$ relative to $t=780^{\circ} \mathrm{C}$ & $\begin{array}{c}\text { y6: } t=1100^{\circ} \mathrm{C} \text { relative } \\
\text { to } t=780^{\circ} \mathrm{C}\end{array}$ \\
\hline $\mathrm{y} 7: t=1100^{\circ} \mathrm{C}$ & $\mathrm{y} 9: t=1100^{\circ} \mathrm{C}$ & $\mathrm{y} 4: t=1100^{\circ} \mathrm{C}$ & $\begin{array}{c}\mathrm{y} 7: w=1120 \mathrm{rad} \cdot \mathrm{s}^{-1}, t=1100^{\circ} \mathrm{C} \\
\text { relative to } w=1120 \mathrm{rad} \cdot \mathrm{s}^{-1} \\
t=780^{\circ} \mathrm{C}\end{array}$ & $\begin{array}{c}\text { y8: } w=1120 \mathrm{rad} \cdot \mathrm{s}^{-1} \\
t=1100^{\circ} \mathrm{C} \text { relative to } \\
w=1120 \mathrm{rad} \cdot \mathrm{s}^{-1} \\
t=780^{\circ} \mathrm{C}\end{array}$ \\
\hline $\begin{array}{l}\text { y8: } w=1120 \mathrm{rad} \cdot \mathrm{s}^{-1}, \\
t=1100^{\circ} \mathrm{C}\end{array}$ & $\begin{aligned} \mathrm{y} 10: w & =1120 \mathrm{rad} \cdot \mathrm{s}^{-1} \\
t & =1100^{\circ} \mathrm{C}\end{aligned}$ & $\begin{array}{c}\mathrm{y} 5: w=1120 \mathrm{rad} \cdot \mathrm{s}^{-1}, \\
t=780^{\circ} \mathrm{C}\end{array}$ & - & - \\
\hline $\mathrm{y} 11: w=1250 \mathrm{rad} . \mathrm{s}^{-1}$ & $\mathrm{y} 13: w=1250 \mathrm{rad} . \mathrm{s}^{-1}$ & $\begin{aligned} \mathrm{y} 6: w & =1120 \mathrm{rad} \cdot \mathrm{s}^{-1}, \\
t & =1100^{\circ} \mathrm{C}\end{aligned}$ & - & - \\
\hline $\begin{array}{l}y 12: w=1250 \mathrm{rad} \cdot \mathrm{s}^{-1} \\
t=780^{\circ} \mathrm{C}\end{array}$ & $\begin{array}{c}\mathrm{y} 14: w=1250 \mathrm{rad} \cdot \mathrm{s}^{-1} \\
t=780^{\circ} \mathrm{C}\end{array}$ & 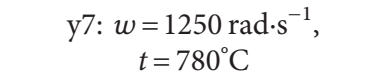 & - & - \\
\hline
\end{tabular}

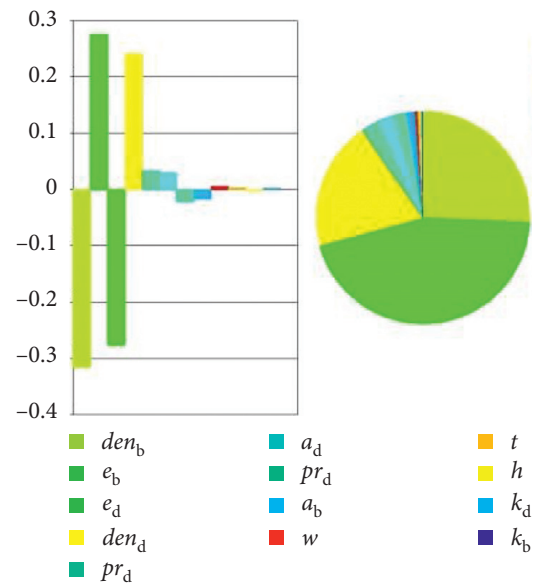

(a)

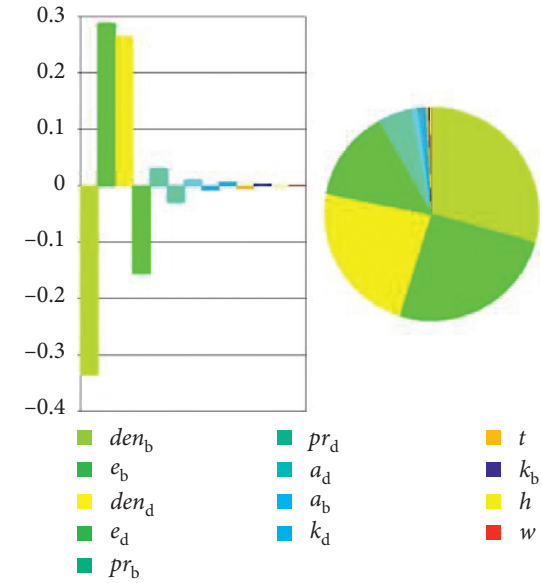

(b)

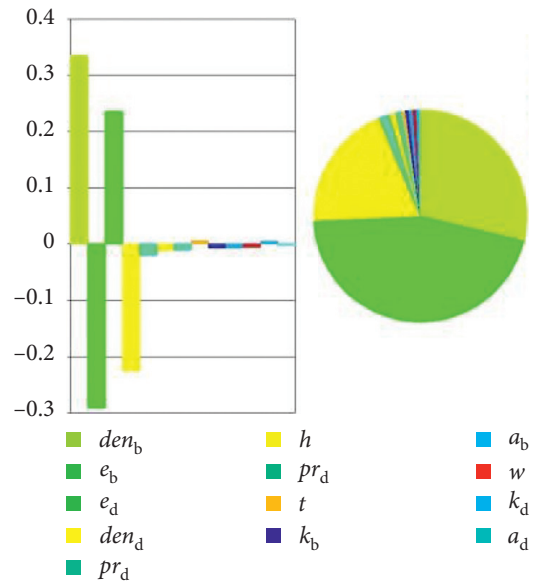

(c)

FIGURE 11: Sensitivity of the modal shape amplitudes for MTB. (a) Sensitivity of DAs. (b) Sensitivity of SAs. (c) Sensitivity of SEAs.

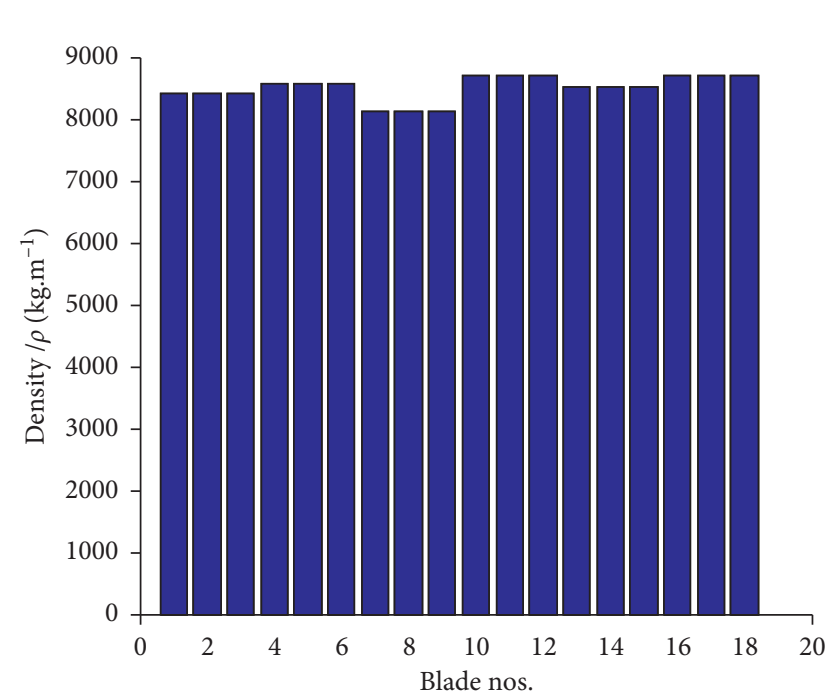

(a)



(b)

FIGURE 12: Intentionally mistuned design. (a) Density of blades. (b) Elastic modulus of blades. 


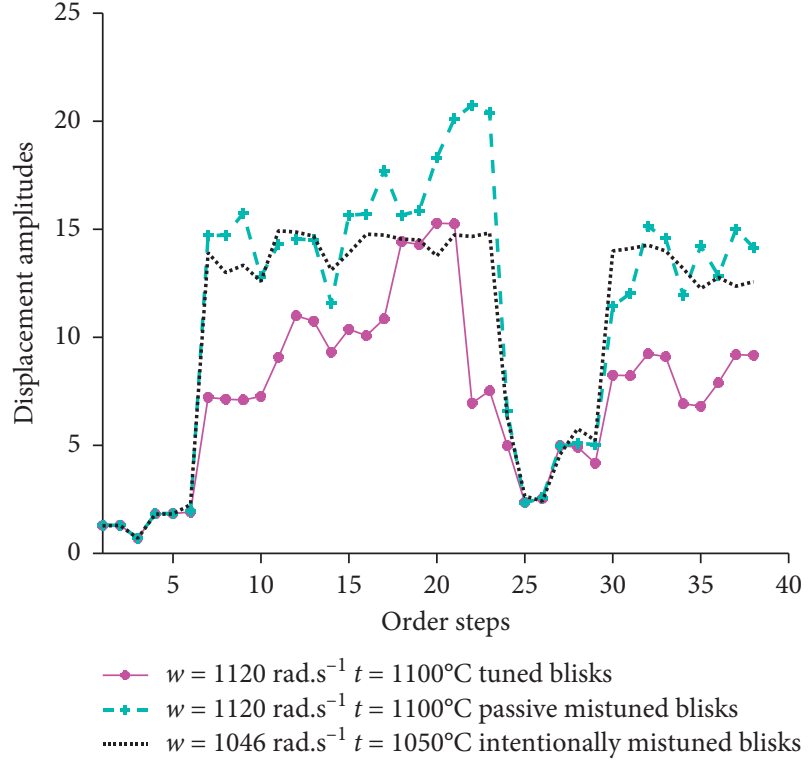

(a)

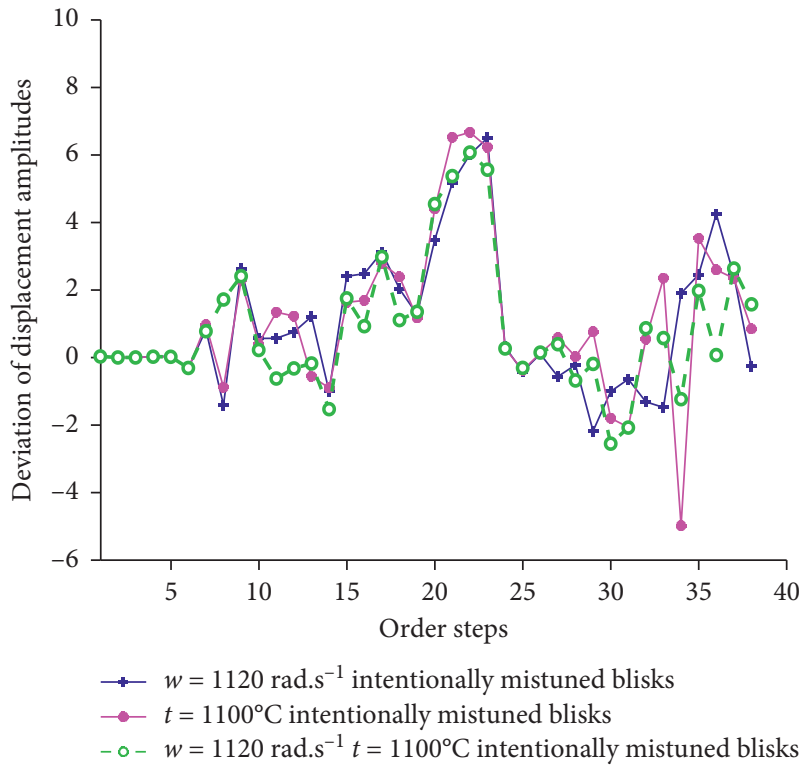

(c)



(b)

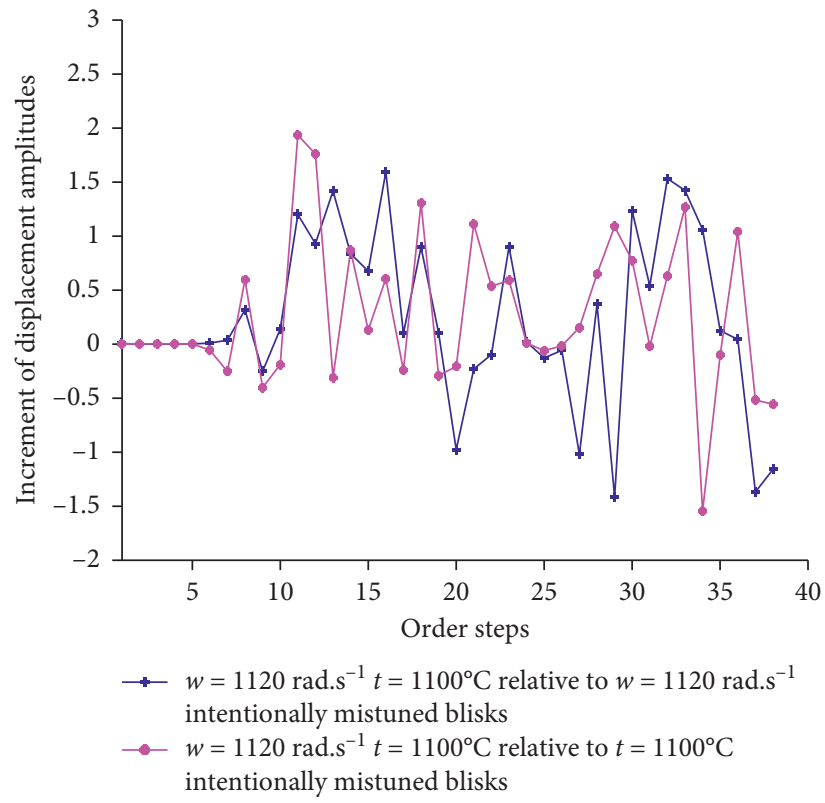

(d)

Figure 13: Displacement amplitudes of intentionally MTB. (a) DAs of tuning, passive, and intentional mistuning. (b) DAs of intentional mistuning. (c) Deviation of DAs. (d) Increment of DAs.

frequency while by temperature in high-order frequency. Although the values significantly increase, they are stable dominated by blade vibration, which means the reliability of bladed disk enhances. Figures 13(c) 15(c) show that deviations of DAs, SAs, and SEAs in intentional mistuning relative to those of in passive mistuning in centrifugal force and thermal load are closer to those of only centrifugal force considered in the first vibration period and closer to those of only thermal load considered in the second vibration period. Figures 13(d) 15(d) reveal that the increments of DAs SAs and SEAs in intentional mistuning relative to in passive mistuning have changed much in the second vibration period than in the first vibration period, the main reason is that the change in passive mistuning is larger than in intentional mistuning.

It is seen from Figures 13-15 that the fluctuation of modal shape amplitudes is reduced and becomes smooth and steady of intentionally MTB; that is, the response is less sensitive to the variables and the robustness is improved. 

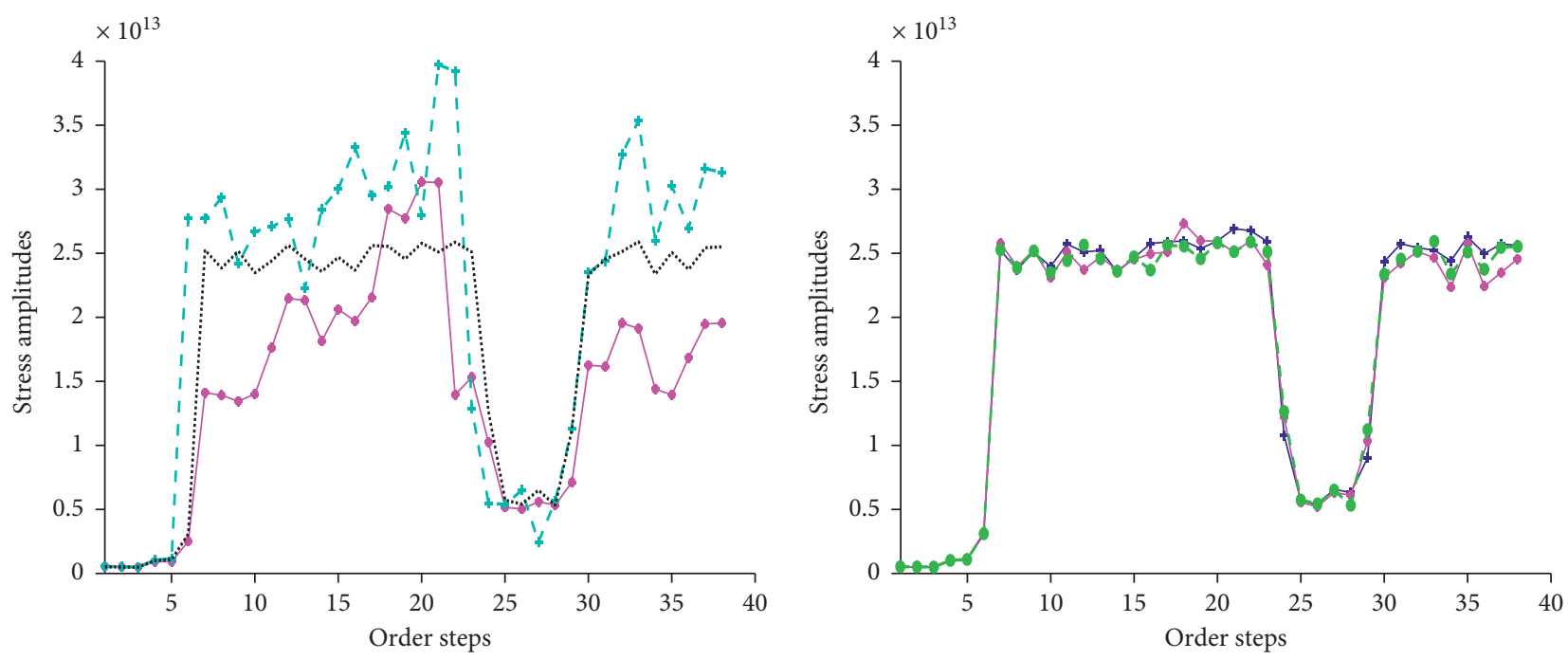

$\rightarrow w=1120 \mathrm{rad} . \mathrm{s}^{-1} t=1100^{\circ} \mathrm{C}$ tuned blisks

- + $w=1120 \mathrm{rad} . \mathrm{s}^{-1} t=1100^{\circ} \mathrm{C}$ passive mistuned blisks

........ $w=1120 \mathrm{rad} . \mathrm{s}^{-1} t=1100^{\circ} \mathrm{C}$ intentionally mistuned blisks

(a)

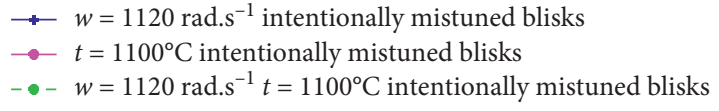

- $w=1120 \mathrm{rad} . \mathrm{s}^{-1} t=1100^{\circ} \mathrm{C}$ intentionally mistuned blisks

(b)
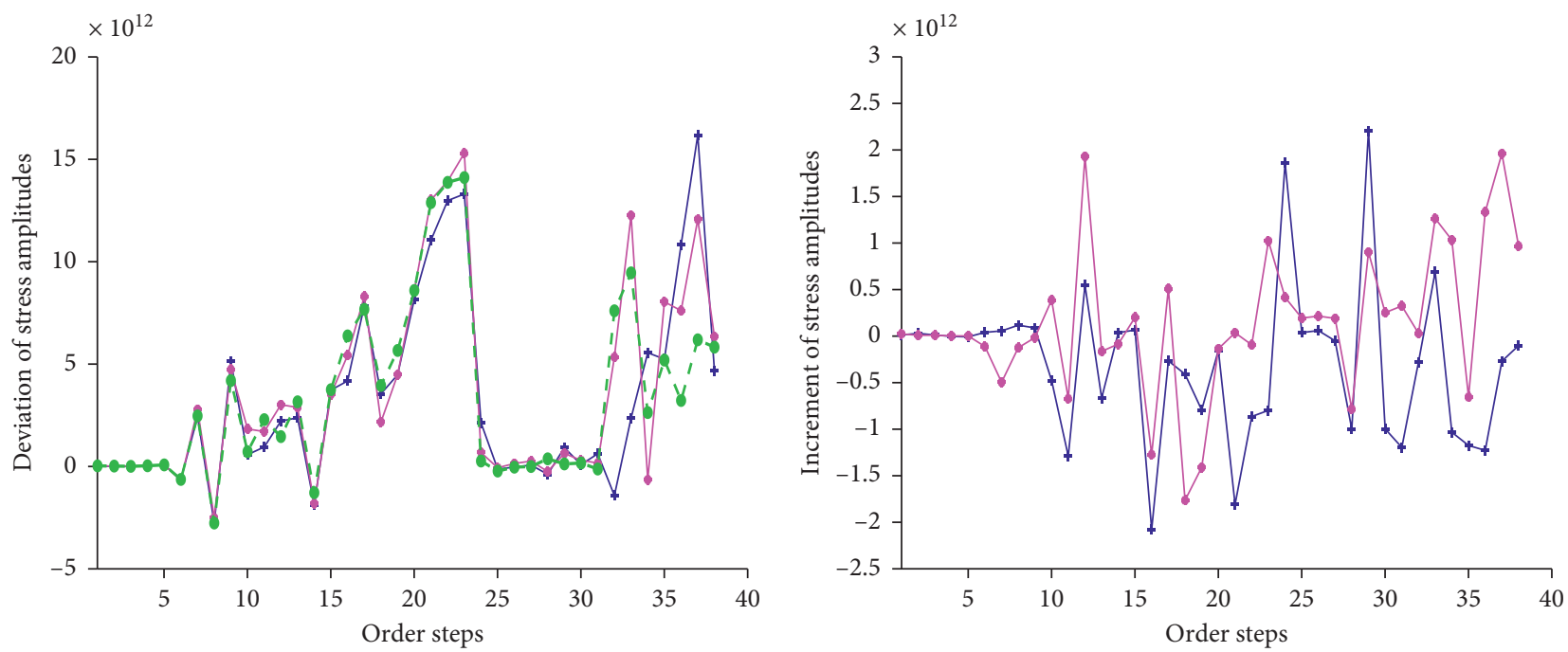

+— $w=1120 \mathrm{rad} . \mathrm{s}^{-1}$ intentionally mistuned blisks

$\rightarrow t=1100^{\circ} \mathrm{C}$ intentionally mistuned blisks

- $-w=1120 \mathrm{rad} . \mathrm{s}^{-1} t=1100^{\circ} \mathrm{C}$ intentionally mistuned blisks

$\rightarrow w=1120 \mathrm{rad} . \mathrm{s}^{-1} t=1100^{\circ} \mathrm{C}$ relative to $w=1120 \mathrm{rad} . \mathrm{s}^{-1}$ intentionally mistuned blisks

$\rightarrow w=1120 \mathrm{rad} \cdot \mathrm{s}^{-1} t=1100^{\circ} \mathrm{C}$ relative to $t=1100^{\circ} \mathrm{C}$ intentionally mistuned blisks

(c)

(d)

FIgURE 14: Stress amplitudes of intentionally MTB. (a) SAs of tuning, passive, and intentional mistuning. (b) SAs of intentional mistuning. (c) Deviation of SAs. (d) Increment of SAs. 


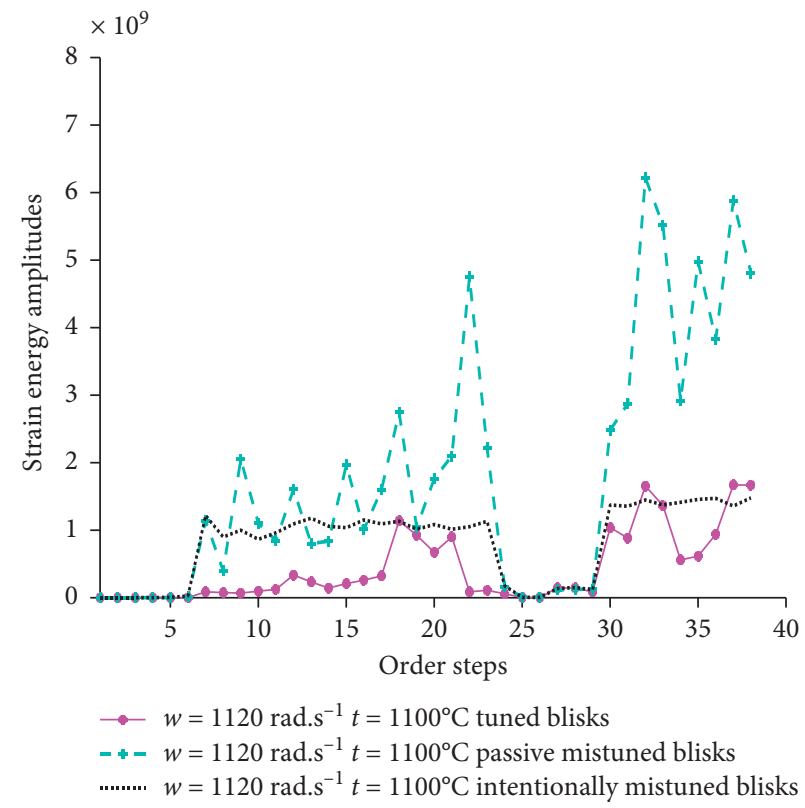

(a)



(c)

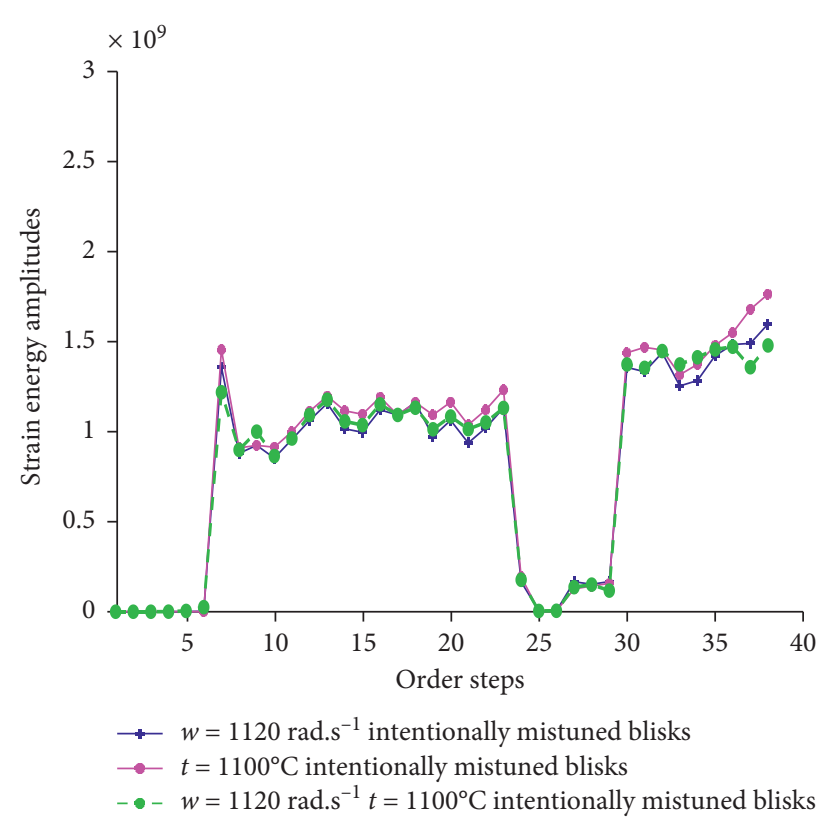

(b)

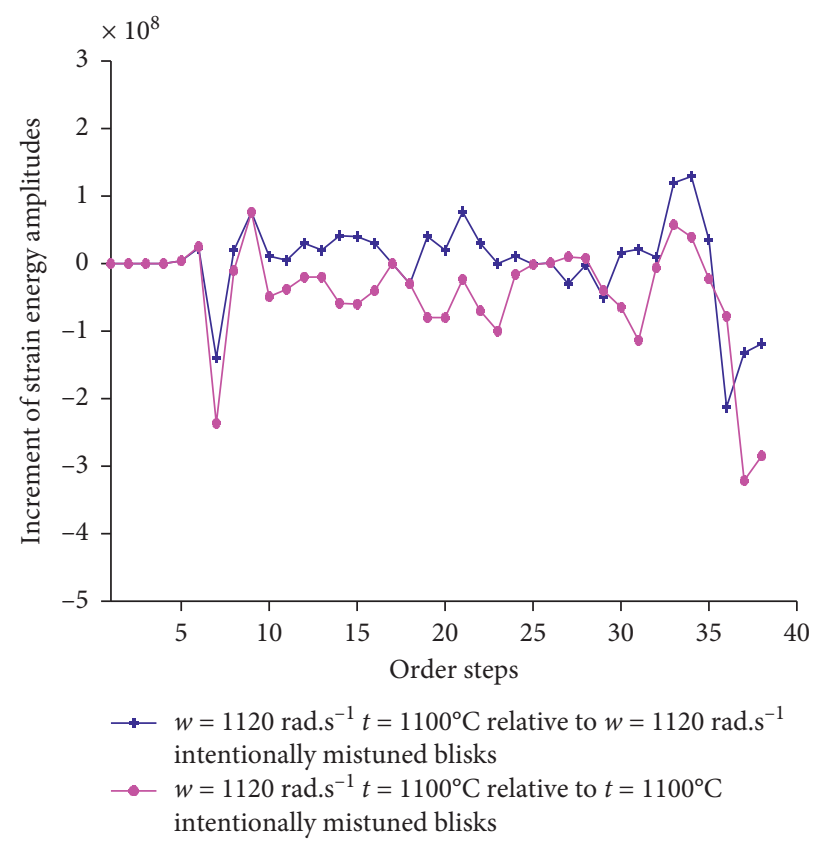

(d)

FIgURE 15: Strain energy amplitudes of intentional MTB. (a) SEAs of tuning, passive, and intentional mistuning. (b) SEAs of intentional mistuning. (c) Deviation of SEAs. (d) Increment of SEAs.

\section{Conclusions}

An improved component modal synthesis-based nonproportional mistuning method (ICMS-NPMM) is proposed, which considers elasticity modulus, thickness, and density of blades, as well as centrifugal force and thermal load. Based on this theory, the reduced-order FEM of turbine blisks is established and its DOFs are obviously reduced. The investigation can obtain the following conclusions:
(1) The intentional mistuning is adopted, and MTB is decomposed into mistuned blade and tuned disk. Meanwhile, the substructures are improved and synthesized. The reduced-order FEM is established by ICMS-NPMM, which can be used to analyze small or large mistuning of turbine blisks.

(2) The DAs, SAs, and SEAs of first 38 order frequencies of the passive MTB are investigated using ICMSNPMM and analyzed the influence of mistuning, centrifugal force, and thermal load on them. This 
investigation indicates that the modal shape amplitudes in low-order frequency are caused by disk or blade-disk coupling bending vibration, and they in high-order frequency are caused by blade torsional vibration or blade-disk coupling torsional vibration, which shows that the destructive of torsional vibration is more severe and the mistuning is more sensitive to the vibration governed by blades.

(3) Based on the investigation of the modal shape amplitudes in passive mistuning, the intentionally mistuned design is performed to improve the robustness and decrease the destructiveness of flutter for MTB. The modal shape amplitudes are reduced significantly by intentionally mistuned design, and the change is smooth and steady. Furthermore, the amplitude of fluctuation governed by blades decreases distinctly and become comparatively smooth and steady. Particularly, the modal shape amplitudes are more stable and less fluctuant, and the sensitivity of output response to input variables is reduced when the intentional MTB enters into the second vibration period.

\section{Data Availability}

The data used to support the findings of this study are currently under embargo while the research findings are commercialized. Requests for data, (6/12 months) after publication of this article, will be considered by the corresponding author.

\section{Conflicts of Interest}

The authors declare that there are no conflicts of interest regarding the publication of this paper.

\section{Acknowledgments}

The authors gratefully acknowledge the financial supports for this research from the National Key R\&D Plan Project (grant no. 2017YFB1301300), National Natural Science Foundation of China (grant nos. 11772011 and 11902220), National Natural Science Foundation of Hebei Province (grant no. E2020202217), and Open Research Fund for Key Laboratory of National Defense Science and Technology for Particle Transport and Enrichment Technology.

\section{References}

[1] S. Y. Deng, J. Y. Yao, L. L. Wang, J. Q. Xin, and N. Hu, "Comparative studies of surrogate models for response analysis of mistuned bladed disks," International Journal of Computational Methods, vol. 17, no. 10, Article ID 2050012, 2020.

[2] X. P. Niu, R. Z. Wang, D. Liao, S. P. Zhu, X. C. Zhang, and B. Keshtegar, "Probabilistic modeling of uncertainties in fatigue reliability analysis of turbine bladed disks," International Journal of Fatigue, vol. 142, Article ID 105912, 2021.

[3] C. Lu, Y.-W. Feng, C.-W. Fei, and S.-Q. Bu, "Improved decomposed-coordinated kriging modeling strategy for dynamic probabilistic analysis of multicomponent structures," IEEE Transactions on Reliability, vol. 69, no. 2, pp. 440-457, 2020.

[4] H. Zhang, H. Yuan, W. Yang, and T. Zhao, "Study on localization influences of frequency veering on vibration of mistuned bladed disk," Journal of Mechanical Science and Technology, vol. 31, no. 11, pp. 5173-5184, 2017.

[5] M. Shadmani, R. Tikani, and S. Ziaei-Rad, "On using a distributed-parameter model for modal analysis of a mistuned bladed disk rotor and extracting the statistical properties of its in-plane natural frequencies," Journal of Sound and Vibration, vol. 438, pp. 324-343, 2019.

[6] T. Giersch, P. Hoenisch, B. Beirow, and A. Kuehhorn, "Forced response analyses of mistuned radial inflow turbines," Journal of Turbomachinery, vol. 135, no. 3, Article ID 031034, 2013.

[7] C. P. Zang, Y. Q. Tan, and E. P. Petrov, "Analysis of forced response for bladed disks mistuned by material anisotropy orientation scatters," Journal of Engineering for Gas Turbines and Power, vol. 140, no. 2, Article ID 022503, 2018.

[8] M. Bonhage, J. T. Adler, C. Kolhoff et al., "Transient amplitude amplification of mistuned structures: an experimental validation," Journal of Sound and Vibration, vol. 436, pp. 236-252, 2018.

[9] N. Jamia, P. Rajendran, S. El-Borgi, and M. I. Friswell, "Mistuning identification in a bladed disk using wavelet packet transform," ACTA Mechanica, vol. 229, no. 3, pp. 1275-1295, 2018.

[10] H. Ma, Y. Lu, Z. Wu, X. Tai, and B. C. Wen, "Vibration response analysis of a rotational shaft-disk-blade system with blade-tip rubbing," International Journal of Mechanical Sciences, vol. 107, pp. 110-125, 2016.

[11] M. S. Kotambkar and A. Chatterjee, "Modeling and investigation of modal properties under crack induced mistuning in a turbine blade packet," Journal of Vibration Engineering \& Technologies, vol. 4, no. 4, pp. 335-348, 2016.

[12] J. Yuan, F. Scarpa, B. Titurus, G. Allegri, S. Patsias, and R. Rajasekaran, "Novel frame model for mistuning analysis of bladed disk systems," Journal of Vibration and Acoustics, vol. 139, no. 3, Article ID 031016, 2017.

[13] T. Klauke, U. Strehlau, and A. Kuehhorn, "Integer frequency veering of mistuned blade integrated disks," Journal of Turbomachinery, vol. 135, no. 6, Article ID 061004, 2013.

[14] M. G. Salas, R. Bladh, H. Martensson, P. Petrie-Repar, and T. Fransson, "Forced response analysis of a mistuned compressor blisk comparing three different reduced order model approaches," Journal of Engineering for Gas Turbines and Power, vol. 139, no. 6, Article ID 062501, 2017.

[15] S. H. Baek and B. I. Epureanu, "Reduced-order models of blisks with small geometric mistuning," Journal of Vibration and Acoustics, vol. 139, no. 4, Article ID 041003, 2017.

[16] B. Bai, H. Li, W. Zhang, and Y. C. Cui, "Application of extremum response surface method-based improved substructure component modal synthesis in mistuned turbine bladed disk," Journal of Sound and Vibration, vol. 472, Article ID 115210, 2020.

[17] C. Lu, C. W. Fei, H. Li, H. T. Liu, and L. Q. An, "Moving extremum surrogate modeling strategy for dynamic reliability estimation of turbine blisk with multi-physics fields," Aerospace Science and Technology, vol. 106, Article ID 106112, 2020.

[18] C. Maravilla, S. Yepifanov, and I. Loboda, "Improvement of turbine blade lifetime assessment by more accurate estimation of the thermal boundary conditions," Advances in Mechanical Engineering, vol. 9, no. 4, 2017. 
[19] A. Picou, E. Capiez-Lernout, C. Soize, and M. Mbaye, "Robust dynamic analysis of detuned-mistuned rotating bladed disks with geometric nonlinearities," Computational Mechanics, vol. 65 , no. 3, pp. 711-730, 2020.

[20] D. Amirante, N. J. Hills, and C. J. Barnes, "A moving mesh algorithm for aero-thermo-mechanical modelling in turbomachinery," International Journal for Numerical Methods in Fluids, vol. 70, no. 9, pp. 1118-1138, 2012.

[21] J. Jarrett, T. Ghisu, G. Parks, and P. Clarkson, "Robust design optimization of gas turbine compression systems," Journal of Propulsion and Power, vol. 27, pp. 282-295, 2011.

[22] L. C. Saikia and S. K. Sahu, "Automatic generation control of a combined cycle gas turbine plant with classical controllers using firefly algorithm," International Journal of Electrical Power \& Energy Systems, vol. 53, pp. 27-33, 2013.

[23] E. Mohammadi and M. Montazeri-Gh, "A fuzzy-based gas turbine fault detection and identification system for full and part-load performance deterioration," Aerospace Science and Technology, vol. 46, pp. 82-93, 2015.

[24] A. Natarajan and D. Verelst, "Outlier robustness for wind turbine extrapolated extreme loads," Wind Energy, vol. 15, no. 2, pp. 679-697, 2012.

[25] X. Y. Kong, J. Xiao, D. H. Liu, J. Z. Wu, C. S. Wang, and Y. Shen, "Robust stochastic optimal dispatching method of multi-energy virtual power plant considering multiple uncertainties," Applied Energy, vol. 279, Article ID 115707, 2020.

[26] Z. F. Qin, X. Q. Bai, and X. Y. Su, "Robust stochastic dynamic optimal power flow model of electricity-gas integrated energy system considering wind power uncertainty," Complexity, vol. 2020, Article ID 8879906, 11 pages, 2020.

[27] W. Wang, S. Caro, F. Bennis, R. Soto, and B. Crawford, "Multi-objective robust optimization using a post optimality sensitivity analysis technique: application to a wind turbine design," Journal of Mechanical Design, vol. 137, no. 1, Article ID 011403, 2015.

[28] Y. Q. Zheng, H. D. Ma, J. F. Wei, and K. Zhu, "Robust optimization for composite blade of wind turbine based on kriging model," Advanced Composites Letters, vol. 29, 2020.

[29] A. J. Keane, "Cokriging for robust design optimization," AIAA Journal, vol. 50, no. 11, pp. 2351-2364, 2012.

[30] Z. Yang, M. Peng, Y. Cao, and L. Zhang, "A new multi-objective reliability-based robust design optimization method," Computer Modeling in Engineering \& Sciences, vol. 98, pp. 409-442, 2014.

[31] P. Kai, F. Ding, Y. Fan, F. Qiang, and L. Yong, "Active generalized predictive control of turbine tip clearance for aero-engines," Chinese Journal of Aeronautics, vol. 26, pp. 1147-1155, 2013.

[32] B. Beirow, F. Figaschewsky, A. Kühhorn, and A. Bornhorn, "Vibration analysis of an axial turbine blisk with optimized intentional mistuning pattern," Journal of Sound and Vibration, vol. 442, pp. 11-27, 2019.

[33] B. Beirow, F. Figaschewsky, A. Kuehhorn, and A. Bornhorn, "Modal analyses of an axial turbine blisk with intentional mistuning," Journal of Engineering for Gas Turbines and Power, vol. 140, Article ID 012503, 2018.

[34] X. Kan, Z. Xu, B. Zhao, and J. Zhong, "Effect of Coriolis force on forced response magnification of intentionally mistuned bladed disk," Journal of Sound and Vibration, vol. 399, pp. 124-136, 2017.

[35] H. Schoenenborn and M. de Vries, "Aeroelasticity at reversed flow conditions-part iii: reduction of surge loads by means of intentional mistuning," Journal of Turbomachinery, vol. 135, Article ID 041009, 2013.
[36] D. Liao, S. P. Zhu, B. Keshtegar, G. Qian, and Q. Y. Wang, "Probabilistic framework for fatigue life assessment of notched components under size effects," International Journal of Mechanical Sciences, vol. 181, Article ID 105685, 2020.

[37] D. Yoo, I. Lee, and J. Tang, "Reliability-oriented optimal design of intentional mistuning for a bladed disk with random and interval uncertainties," Engineering Optimization, vol. 49, no. 5, pp. 796-814, 2017.

[38] C. W. Fei, H. Li, H. T. Liu, C. Lu, and B. Keshtegar, "Multilevel nested reliability-based design optimization with hybrid intelligent regression for operating assembly relationship," Aerospace Science and Technology, vol. 103, Article ID 105906, 2020.

[39] Y. Bhartiya and A. Sinha, "Reduced order modeling of a bladed rotor with geometric mistuning via estimated deviations in mass and stiffness matrices," Journal of Engineering for Gas Turbines and Power, vol. 135, Article ID 052501, 2013.

[40] J. Yao, J. Wang, and Q. Li, "Improved modal localization and excitation factors for understanding mistuned bladed disk response," Journal of Propulsion and Power, vol. 27, no. 1, pp. 50-60, 2011.

[41] B. Bai, W. Zhang, and B. T. Li, "Interval non-probabilistic analysis based on a dynamic substructural collaborative finiteelement method for aeroengine blisks," Journal of Aerospace Engineering, vol. 30, no. 5, Article ID 04017047, 2017. 